\title{
Development of an Open-source Alloy selection and Lifetime assessment tool for structural components in CSP
}

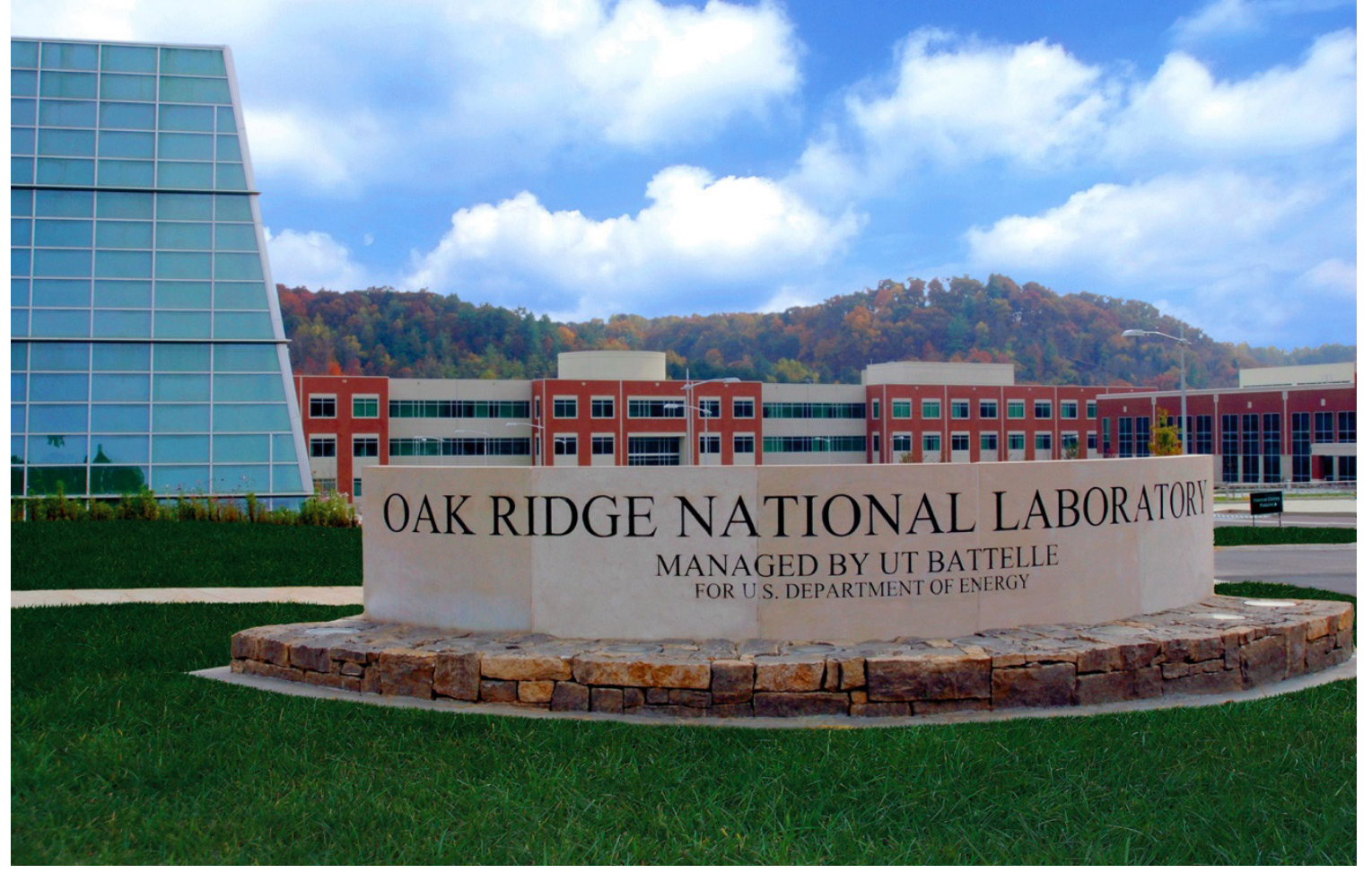

Rishi Pillai

Marie Romedenne

Sangkeun Lee

January 2022 


\title{
DOCUMENT AVAILABILITY
}

Reports produced after January 1, 1996, are generally available free via OSTI.GOV.

Website www.osti.gov

Reports produced before January 1, 1996, may be purchased by members of the public from the following source:

\author{
National Technical Information Service \\ 5285 Port Royal Road \\ Springfield, VA 22161 \\ Telephone 703-605-6000 (1-800-553-6847) \\ TDD 703-487-4639 \\ Fax 703-605-6900 \\ E-mail info@ntis.gov \\ Website http://classic.ntis.gov/
}

Reports are available to US Department of Energy (DOE) employees, DOE contractors, Energy Technology Data Exchange representatives, and International Nuclear Information System representatives from the following source:

Office of Scientific and Technical Information

PO Box 62

Oak Ridge, TN 37831

Telephone 865-576-8401

Fax 865-576-5728

E-mail reports@osti.gov

Website https://www.osti.gov/

This report was prepared as an account of work sponsored by an agency of the United States Government. Neither the United States Government nor any agency thereof, nor any of their employees, makes any warranty, express or implied, or assumes any legal liability or responsibility for the accuracy, completeness, or usefulness of any information, apparatus, product, or process disclosed, or represents that its use would not infringe privately owned rights. Reference herein to any specific commercial product, process, or service by trade name, trademark, manufacturer, or otherwise, does not necessarily constitute or imply its endorsement, recommendation, or favoring by the United States Government or any agency thereof. The views and opinions of authors expressed herein do not necessarily state or reflect those of the United States Government or any agency thereof. 
Corrosion Science \& Technology

Physical Sciences Directorate

\section{DEVELOPMENT OF AN OPEN-SOURCE ALLOY SELECTION AND LIFETIME ASSESSMENT TOOL FOR STRUCTURAL COMPONENTS IN CSP}

R. Pillai, M. Romedenne, S.M. Lee

January 2022

Prepared by OAK RIDGE NATIONAL LABORATORY

Oak Ridge, TN 37831

managed by

UT-BATTELLE LLC

for the

US DEPARTMENT OF ENERGY

under contract DE-AC05-00OR22725 


\section{Final Project Report}

\begin{tabular}{|c|c|c|}
\hline a. Federal Agency & \multicolumn{2}{|c|}{ Department of Energy } \\
\hline b. Award Number & \multicolumn{2}{|l|}{37370} \\
\hline c. Project Title & \multicolumn{2}{|c|}{$\begin{array}{l}\text { Development of an Open-source Alloy selection and } \\
\text { Lifetime assessment tool for structural components } \\
\text { in CSP }\end{array}$} \\
\hline d. Principal Investigator & \multicolumn{2}{|c|}{$\begin{array}{l}\text { Rishi Pillai } \\
\text { Research Staff } \\
\text { Email: pillairr@ornl.gov } \\
\text { Phone: 865-574-4451 }\end{array}$} \\
\hline e. Business Contact & \multicolumn{2}{|c|}{$\begin{array}{l}\text { Claus Daniel } \\
\text { Program Manager } \\
\text { Email: danielc@ornl.gov } \\
\text { Phone: 865-241-9521 } \\
\end{array}$} \\
\hline f. Submission Date & \multicolumn{2}{|l|}{ December 31, 2021} \\
\hline g. DUNS Number & \multicolumn{2}{|l|}{ DUNS Number } \\
\hline h. Recipient Organization & \multicolumn{2}{|c|}{ Oak Ridge National Laboratory } \\
\hline i. Project Period & Start: $10 / 01 / 2020$ & End: 09/30/2021 \\
\hline j. Reporting Period & Start: $10 / 01 / 2020$ & End: 09/30/2021 \\
\hline k. Report Term or Frequency & \multicolumn{2}{|l|}{ annual } \\
\hline I. Submitting Official Signature & \multicolumn{2}{|c|}{ Electronic signature (i.e., Adobe Acrobat) } \\
\hline
\end{tabular}




\section{Contents}

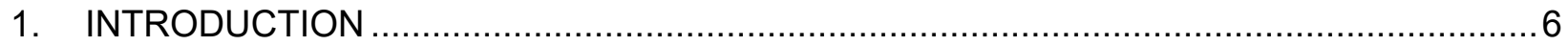

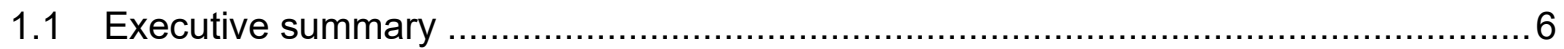

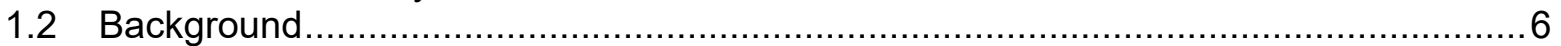

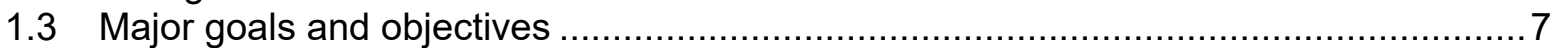

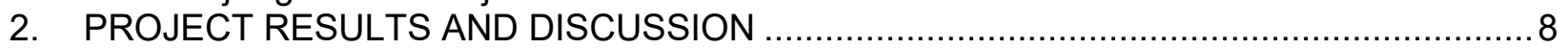

2.1.1 Task 1:Determine framework for model development ............................... 8

2.1.2 Task 2: Model development .............................................................19

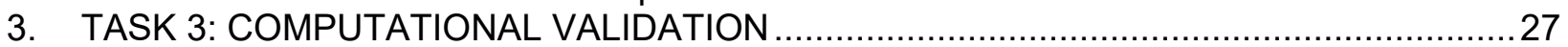

3.1 Subtask 3.1: Compare Calculated and Measured $\mathrm{Cr}$ Concentration at Oxide-

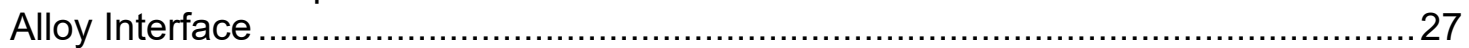

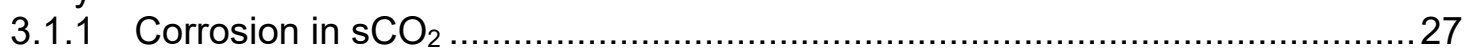

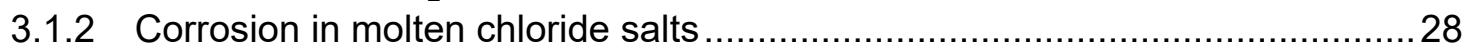

4. TASK 4: GENERATE SCREENING PROTOCOL FOR CURRENTLY IMMATURE

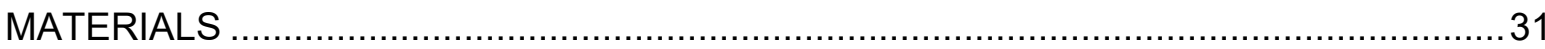

4.1.1 Subtask 4.1: Review existing literature data for one chosen sample

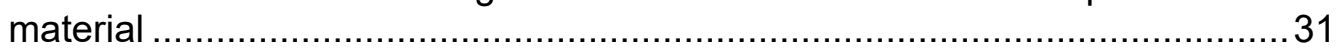

4.1.2 Subtask 4.2: Identify key degradation mechanisms of chosen material .......... 32

4.1.3 Subtask 4.3: Obtain information on required model parameters to describe degradation and predict lifetime of chosen material ..........................33

5. TASK 5: PROVIDE ACCESSIBILITY TO MODELING TOOL ……..............................36

5.1.1 Subtask 5.1 Provide developed tool for testing to user (ORNL) …….............36

5.1.2 Subtask 5.2 Upload developed tool to GITHUB ............................................36 


\section{Introduction}

\subsection{Executive summary}

Lack of sufficient data on high temperature mechanical and corrosion behavior of structural materials is a huge barrier in the technological maturity of current and future Concentrating Solar Power (CSP) technologies. Rapid development and selection of materials cannot be achieved by expensive and time-consuming acquisition of experimental data. The goal of the proposed work is development of an open-source alloy selection and lifetime prediction tool that will integrate validated physics-based models to describe influence of temperature, alloy composition, environment and component geometry (thickness) on mechanical and corrosion behavior of $\mathrm{Ni}$ and Fe-based alloys employed in molten salts $/ \mathrm{sCO}_{2}$ heat exchangers.

This one-year project leveraged the extensive dataset on the creeplcorrosion behavior of candidate materials generated at ORNL through past projects and input from current collaborations with industrial partners. Based on previous experience and the feedback provided by industry (Brayton Energy and Echogen), three candidate materials of interest, Ni-based alloys $740 \mathrm{H}, 282$ and 625 and application-specific operating conditions (max. temperature of $730{ }^{\circ} \mathrm{C}$ and stress of $150 \mathrm{MPa}$ ) were identified for the heat exchanger. An extensive corrosion and creep dataset was assimilated for the relevant operating conditions and was supported by detailed characterization of about 100 metallographic cross-sections. The corrosion dataset consisted of scanning electron microscopy images (secondary electron and backscatter electron), measured concentration profiles of alloying elements using energy dispersive X-ray spectroscopy (EDS), widths of denuded zones (dissolution of strengthening phases) and depths of attack in molten $\mathrm{KCl}-\mathrm{MgCl}_{2}$ mixtures using image analyses. The creep dataset comprised of creep rupture data and creep strain curves (for $740 \mathrm{H}$ and 282 ).

Coupled thermodynamic-kinetic microstructure-based models were employed to predict the stress-corrosion induced compositional and phase evolutions in the alloy during operation under the identified operating conditions. Reduced order models were developed from advanced physics-based models and were integrated in a user-friendly alloy selection tool. The corrosion model was able to predict the time to a critical $\mathrm{Cr}$ concentration at the oxide/alloy interface (chemical lifetime) within $\pm 10 \%$ ( 1 standard deviation) of typical statistical variation in corrosion tests and EDS measurement errors $( \pm 0.5 \mathrm{wt} \%)$. The initial scope of the project was limited to predict creep rupture times (Larson-Miller parameter). Based on the input provided by industry, the mechanical lifetime of the heat exchanger is governed by accumulated creep strains $(2 \%)$ rather than creep rupture. To be able to predict the times to specific creep strains, a more extensive creep model development was undertaken largely beyond the initial scope of the project. The continuum damage mechanics creep model was able to predict times to $2 \%$ creep strain, $t_{2} \%$ with an accuracy of $\pm 500 \mathrm{~h}$.

Ultimately, a screening protocol for $\mathrm{SiC}$ was generated to demonstrate the pathway for integration of one of the currently immature materials from a commercial adoption standpoint in the current material evaluation tool. The modeling tool developed here is accessible to the science community and stakeholders and lays the foundation for methods that will enable a rapid evaluation of optimum materials for CSP applications and reliable prediction of material degradation thereby considerably reducing operational costs, improving reliability and increasing overhaul intervals. However, the complete potential of such a tool to include a wider range of materials and test conditions can only be realized with a more concentrated combined experimental-characterization-computation effort.

\subsection{Background}

Integrating a supercritical $\mathrm{CO}_{2}$ power cycle with concentrating solar power employing thermal energy storage with chloride salts is considered a key driver in achieving the efficiency 
goal of $50 \%$ by operating at temperatures over $700^{\circ} \mathrm{C}$ to achieve the future levelized cost of electricity (LCOE) SunShot 2030 targets [1, 2]. However, these technologies all face a critical limitation, namely the lack of advanced materials that can mitigate degradation due to supercritical $\mathrm{CO}_{2}$ and molten salts and ensure safe, successful operation of structural components at temperatures over $700^{\circ} \mathrm{C}[3,4]$. Material selection for CSP technologies based on experiments has to replicate the dynamic operating conditions of thermal cycling under harsh environments and testing for expected lifetimes of about 100kh can only be achieved through an extensive and costly research effort. Models can accelerate alloy development by aiding in material evaluation but need to integrate the extremely complex physics of the processes (environment-alloy interactions, transport mechanisms through the surface corrosion layers) to allow a reliable prediction of key design parameters such as corrosion rates and deterioration of mechanical properties.

Existing efforts to predict degradation of candidate structural materials for CSP systems [1-4] have considered the effects of individual damage mechanisms such as creep, fatigue and corrosion. Barua et al. [1] developed a thermomechanical model for a Gen3 CSP solar receiver to evaluate the applicability of different creep-fatigue and ratcheting design methods identified in Section III, Division 5 of the ASME Boiler and Pressure Vessel Code for CSP systems. Three distinct failure modes induced by corrosion in $\mathrm{SCO}_{2}$ were presented by Kung et al. [2] for heat exchangers: (i) reduction in area of flow channels, (ii) exhaustion of $\mathrm{Cr}$ reservoir in the heat exchanger wall and (iii) blockage of flow channels due to exfoliated scales. Based on these failure criteria, a combination of analytical and high fidelity physics-based modeling was employed in [4] to predict the corrosion-induced lifetime of alloys $740 \mathrm{H}, 282$ and 625 .

A few studies have dealt with models to predict corrosion rates of $\mathrm{Ni}$-based alloys during exposure in molten halide salts [5-10]. Models presented in [5-9] are useful tools to predict surface corrosion rates but they do not consider corrosion-driven chemical interactions between alloying elements and transport processes in the alloy. Pillai et al. [10] addressed this shortcoming by employing a coupled thermokinetic model and emphasized the importance of examining alloy chemistry and chemical interactions between alloying elements in more detail.

The current project aimed to address two key gaps in the development of design tools for lifetime assessment of structural materials for CSP systems:

- lack of models that consider influence of corrosion on mechanical properties of candidate materials

- lack of design procedures incorporating high-fidelity physics-based models while still simplifying usage and accessibility to non-domain experts

\subsection{Major goals and objectives}

The goal of the proposed work is developing the first version of an open-source alloy selection and lifetime prediction tool that will integrate validated physics-based models to describe the influence of time, temperature, alloy composition, alloy microstructure, environment and component geometry (thickness) on the mechanical and corrosion behavior of Fe- and Ni-based candidate materials for a molten salt $\mathrm{sCO}_{2}$ heat exchanger. Furthermore, the work will generate a pathway for screening currently immature materials with the developed tool.

Within the project, tasks were planned and completed with the ultimate goal of achieving the following project milestones:

1. Dataset for three materials for conditions (three temperatures and stresses) specific to the chosen application and in accordance with physics of material degradation were compiled based on industry feedback

2. Initial version of the model was developed and its functionality was verified by performing test calculations for 1 material at one operating condition to show time evolution of $\mathrm{Cr}$ 
concentration in the alloys undergoing corrosion with $\pm 10 \%$ accuracy within scatter of experimental data.

3. Validation of modeling results by comparing calculated element concentrations and phase fractions with measured concentrations and phase distributions.

4. Generate a review document including information on differences in failure mechanisms and identifying gaps in required experimental data for one chosen material (currently immature).

5. A user-tested tool will be uploaded with an e-link to an open source platform (e.g., GITHUB)

6. A final technical report verified and revised by SETO technology manager will be submitted to osti.gov with an identifiable e-link number

The corresponding tasks were as follows:

- Task 1: Determine framework for model development

- Subtask 1.1 Identifying materials based on chosen application

- Subtask 1.2 Acquire feedback from the industry to review identified applicationspecific conditions

- Subtask 1.3 Compile dataset for 3 identified materials at 3 temperatures

- Task 2: Model development

- Subtask 2.1: Integrate models to describe physics for degradation of identified materials

- Subtask 2.2: Perform test calculations to verify functionality of the model

- Task 3: Computational validation

- Subtask 3.1: Compare calculated and measured $\mathrm{Cr}$ concentration at oxide/alloy interface

- Subtask 3.2: Identify correlation between phase fractions of strengthening phases and LMP

- Task 4: Generate screening protocol for currently immature materials

- Subtask 4.1: Review existing literature data for one chosen sample material ( $\mathrm{SiC}$ )

- Subtask 4.2: Identify key degradation mechanisms of chosen material

- Subtask 4.3: Obtain information on required model parameters to describe degradation and predict lifetime of chosen material

- Task 5: Provide accessibility to modeling tool

- Subtask 5.1: Provide developed tool for testing to user (ORNL)

- Subtask 5.2: Upload developed tool to GITHUB

- Subtask 5.3: Complete final report

2. Project results and discussion Task 1:Determine framework for model development

\subsubsection{Subtask 1.1: Identifying materials based on chosen application}

The first objective of this project was to identify the materials relevant for the chosen application of a molten salt/sCO $\mathrm{SO}_{2}$ heat exchanger. Industry experts (Brayton Energy) were contacted to assist in downselecting three most relevant materials for the heat exchanger designed to operate at temperatures $>550{ }^{\circ} \mathrm{C}$. Ni-based alloys Special Metals $740 \mathrm{H}$ and Haynes 282 are preferred materials for elevated temperatures $\left(>700{ }^{\circ} \mathrm{C}\right)$ specifically for tube heat exchangers. The use of these materials in the plate-fin type of heat exchanger presents certain manufacturing challenges but lifetime assessment of these materials in the plate-fin configuration is extremely relevant from an application perspective. Haynes 230 is an established and recognized ASME material for high temperature service. Special Metals alloy 625 is frequently used in prototyping efforts mainly due to its wider availability alloys in different geometries 
compared to other Ni-based alloys. However, high temperature performance has been observed to vary between different material grades (asterisk in Pressure Vessel Code).

Based on the discussions with the industry experts and the availability of relevant creep and corrosion data (in $\mathrm{sCO}_{2}$ ) at $\mathrm{ORNL}, 740 \mathrm{H}, 282$ and 625 were selected as three materials to be included in the development of the material selection and lifetime assessment tool. The measured chemical composition of these alloys is given in Table 1. These three materials were also identified as key candidate materials for CSP components under the previously conducted project at ORNL (DE-EE0001556). Furthermore, evaluation of the application geometry (sheet form) on the high temperature performance of $740 \mathrm{H}$ and 282 is currently being evaluated in an ongoing project with Brayton Energy (DE EE0008994).

Table 1: Measured chemical composition of the alloys.

\begin{tabular}{|c|c|c|c|c|c|c|c|c|c|c|c|}
\hline Alloy & $\mathbf{F e}$ & $\mathbf{N i}$ & $\mathbf{C r}$ & $\mathbf{A l}$ & $\mathbf{C o}$ & $\mathbf{M o}$ & $\mathbf{N b}$ & $\mathbf{T i}$ & $\mathbf{M n}$ & $\mathbf{S i}$ & $\mathbf{C}$ \\
\hline Haynes 282 & 0.2 & 57.1 & 19.6 & 1.6 & 10.6 & 8.6 & $<0.01$ & 2.2 & 0.02 & 0.04 & 0.059 \\
\hline Inconel $740 \mathrm{H}$ & 0.1 & 49.7 & 24.5 & 1.4 & 20.6 & 0.3 & 1.5 & 1.4 & 0.3 & 0.2 & 0.027 \\
\hline 625 & 4.0 & 61.0 & 21.7 & 0.12 & 0.1 & 8.8 & 3.5 & 0.2 & 0.2 & 0.2 & 0.016 \\
\hline
\end{tabular}

\subsubsection{Subtask 1.2: Acquire feedback from the industry to review identified application- specific conditions}

Brayton Energy and Echogen were contacted to provide feedback on the materials identified for the heat exchanger (Subtask 1.1) and review relevant operating conditions. Furthermore, guidance was requested in specifying the failure criteria for this application to incorporate in the lifetime assessment of the chosen materials. Based on the discussions with Brayton Energy, maximum operating temperatures and allowable stresses for candidate materials employed in heat exchangers and solar receivers with $\mathrm{sCO}_{2}$ as the operating fluid are specified in Table 2. Accumulated creep strain was identified as the primary degradation mechanism and standard practice typically requires creep strains to be less than $2 \%$.

Echogen agreed with the three chosen materials for the chosen temperature range of 700$800^{\circ} \mathrm{C}$. The inclusion of alloy 617 was suggested since the alloy is code-case accepted for diffusion bonded heat exchangers. Additional feedback was provided regarding the potential failure mechanisms for $\mathrm{sCO}_{2}$ heat exchangers as follows:

- Corrosion-induced material loss

- Creep

- Plugging of passages either due to corrosion products from upstream locations or oxide growth in the passages

- Stress concentrations at plate-bonding boundaries

A widely accepted failure criteria for corrosion induced degradation of heat exchanger components could not be ascertained [11, 12]. Within the framework of a previously conducted project at ORNL (DE-EE0001556), various potential corrosion-induced degradation mechanisms were addressed. The critical depletion of $\mathrm{Cr}$ in the alloys $740 \mathrm{H}, 282$ and 625 during exposure in high pressure $\mathrm{sCO}_{2}\left(300\right.$ bar $\mathrm{sCO}_{2}$ ) between $700-800{ }^{\circ} \mathrm{C}$ was employed as corrosion-induced lifetime criteria. Beyond the accepted critical $\mathrm{Cr}$ depletion of $10 \mathrm{wt} \%$ [13, 14] at the interface between the external $\mathrm{Cr}_{2} \mathrm{O}_{3}$ scale and the Ni-based alloy, formation of other faster-growing and non-protective oxides such as $\mathrm{Ni}$-, $\mathrm{Fe}$ - and Ti-rich oxides is expected. Additionally, the loss of sound metal due to corrosion will be critical for thin components since the metal loss can be comparable to the initial component thickness. The calculated metal loss rates from measured 
oxidation rates of $740 \mathrm{H}, 282$ and 625 alloy specimens amounted to about $2-5 \mu \mathrm{m}$ at $750{ }^{\circ} \mathrm{C}$ to about $10-15 \mu \mathrm{m}$ at $800{ }^{\circ} \mathrm{C}$ after exposure for $10,000 \mathrm{~h}$. This might be especially critical for component thicknesses of the order of a few hundred microns expected to demonstrate operational lifetimes of $100,000 \mathrm{~h}$. Furthermore, the growth of oxide scales in small flow channels of $\mathrm{sCO}_{2}$ heat exchangers would result in a reduction of the flow areas of the channels which would consequently lead to pressure drops across the channel. Typically, a $2-5 \%$ reduction in flow area (RFA) is considered acceptable [12].

Table 2: Application specific candidate materials, operating conditions, and corresponding failure mechanisms

\begin{tabular}{|c|c|c|c|c|c|c|c|c|}
\hline Application & $\begin{array}{c}\text { Application } \\
\text { geometry }\end{array}$ & Fluid & $\begin{array}{c}\text { Component } \\
\text { thickness } \\
\text { (mils) }\end{array}$ & $\begin{array}{c}\text { Max. } \\
\text { operating } \\
\text { temperature } \\
\left({ }^{\circ} \mathbf{C}\right)\end{array}$ & $\begin{array}{c}\text { Max. } \\
\text { allowable } \\
\text { stress } \\
(\mathbf{M P a})\end{array}$ & Materials & $\begin{array}{c}\text { Degradation } \\
\text { mechanisms }\end{array}$ & $\begin{array}{c}\text { Failure } \\
\text { criteria }\end{array}$ \\
\hline $\begin{array}{c}\text { Heat } \\
\text { exchanger }\end{array}$ & Plate-fin & $\mathrm{sCO}_{2}$ & 8 & 730 & 60 & $\mathrm{H} 230$ & $\begin{array}{c}\text { Accumulated } \\
\text { Creep-Strain }\end{array}$ & Creep \\
\hline $\begin{array}{c}\text { Heat } \\
\text { Exchanger }\end{array}$ & Plate-fin & $\mathrm{sCO}_{2}$ & $4-20$ & 730 & 150 & $\mathrm{IN740/H} 282$ & $\begin{array}{c}\text { Accumulated } \\
\text { Creep-Strain }\end{array}$ & Creep \\
\hline $\begin{array}{c}\text { Solar } \\
\text { Receiver }\end{array}$ & Plate-fin & $\mathrm{sCO}_{2}$ & $4-20$ & 770 & 65 & $\mathrm{IN740/H} 282$ & $\begin{array}{c}\text { Accumulated } \\
\text { Creep-Strain }\end{array}$ & Creep \\
\hline $\begin{array}{c}\text { Solar } \\
\text { Receiver }\end{array}$ & Tube & $\mathrm{sCO}_{2}$ & 80 & 770 & 65 & $\mathrm{IN740/H282}$ & $\begin{array}{c}\text { Accumulated } \\
\text { Creep-Strain }\end{array}$ & Creep \\
\hline
\end{tabular}

\subsubsection{Subtask 1.3: Compile dataset for 3 identified materials at 3 temperatures}

Based on the feedback provided by Brayton Energy on the choice of candidate materials, operating conditions and failure mechanisms for the selected heat exchanger application and deriving from the experimental data generated at ORNL for corrosion behavior of these materials in $\mathrm{SCO}_{2}$ relevant corrosion and creep data were assimilated. Additionally, existing literature data for the three identified materials in molten chloride salts was compiled. A summary of the type and source of data are in Table 3 and Table 4.

Table 3: Type and sources of assimilated corrosion dataset for $740 \mathrm{H} .282$ and 625.

\begin{tabular}{|c|c|c|c|}
\hline Data type & Alloy & Source & Conditions \\
\hline \multirow{3}{*}{$\begin{array}{l}\text { Parabolic rate constant } \\
\text { for } \mathrm{Cr}_{2} \mathrm{O}_{3} \text { formation }\end{array}$} & $740 \mathrm{H}$ & $\begin{array}{c}\text { ORNL [3] } \\
\text { EPRI [2] } \\
\text { KAIST, OSU, UW [15] }\end{array}$ & \multirow{3}{*}{$\begin{array}{l}>650,700,750,800{ }^{\circ} \mathrm{C} \\
>\text { Times up to } 15,000 \mathrm{~h}\end{array}$} \\
\hline & 282 & $\begin{array}{l}\text { ORNL [3] } \\
\text { EPRI [2] } \\
\text { NETL [16] }\end{array}$ & \\
\hline & 625 & $\begin{array}{c}\text { ORNL [3] } \\
\text { EPRI [2] } \\
\text { NETL [16] } \\
\text { KAIST, OSU, UW [15] }\end{array}$ & \\
\hline Cr concentration profiles & \multirow[b]{2}{*}{$740 \mathrm{H}, 282,625$} & \multirow[b]{2}{*}{ ORNL [3] } & \multirow{2}{*}{$\begin{array}{l}30 \text { metallographic cross- } \\
\text { sections } \\
21 \text { time-temperature } \\
\text { combinations ( } 7 \text { per alloy) }\end{array}$} \\
\hline $\begin{array}{l}\text { Widths of denuded zones of } \\
\text { strengthening phases }\end{array}$ & & & \\
\hline $\begin{array}{l}\text { Depth of attack in chloride } \\
\text { salts }\end{array}$ & $\begin{array}{c}\mathrm{C} 276,600,230 \\
740 \mathrm{H}\end{array}$ & ORNL [17] & $\begin{array}{l}7 \text { metallographic cross- } \\
\text { sections } \\
4 \text { time-temperature } \\
\text { combinations ( } 1 \text { per alloy) }\end{array}$ \\
\hline
\end{tabular}


Essential corrosion data for model development and validation should include measured concentration profiles after exposures at the temperatures of interest $\left(700-800^{\circ} \mathrm{C}\right)$ for different times. For exposures in $\mathrm{sCO}_{2}$, depletion of the alloying element forming the protective oxide can be correlated to the temporal evolution of specific mass change measurements for the three alloys $(740 \mathrm{H}, 282$ and 625), since these alloys have been reported to form compact and protective $\mathrm{Cr}$-rich $\mathrm{Cr}_{2} \mathrm{O}_{3}$ scales up to $800^{\circ} \mathrm{C}$ [2, 4, 18-25]. However, for exposures in molten chloride salts, a combination of the extremely low fraction of species reacting in the boundary regions (a few microns) between phases or molecular regions and nonequilibrium reaction kinetics provide challenges in identifying corrosion products. Furthermore, mass change data is not representative of the compositional changes in the alloy since the corrosion behavior is strongly dependent on the salt chemistry (different purification procedures), use of different container materials (dissimilar capsule/test material combinations) and employing distinct test procedures (capsule/loop tests). The existing corrosion data in the literature for $740 \mathrm{H}$, 282 and 625 in $\mathrm{sCO}_{2}$ and molten chloride salts between $700-800^{\circ} \mathrm{C}$ was assimilated and the specifics will be discussed in the following sections.

Table 4: Type and sources of assimilated creep dataset for $740 \mathrm{H} .282$ and 625.

\begin{tabular}{|c|c|c|c|}
\hline Data type & Alloy & Source & Number of datasets \\
\hline \multirow{2}{*}{$\begin{array}{c}\text { Larson Miller } \\
\text { Parameter }\end{array}$} & $740 \mathrm{H}, 282$ & ORNL,EPRI [26, 27] & 134 stress-temperature combinations \\
\cline { 2 - 4 } & 625 & ANL [28] & 15 stress-temperature combinations \\
\hline Creep strain curves & $740 \mathrm{H}$ & ORNL [29] & 111 creep curves \\
\cline { 2 - 4 } & 282 & ORNL [30] & 60 creep curves \\
\hline Widths of denuded zones & $740 \mathrm{H}, 282$ & ORNL & 45 metallographic cross-sections \\
\hline
\end{tabular}

\section{Corrosion Data in $\mathrm{sCO}_{2}$}

Multiple data sources were reviewed to assimilate the temperature dependence of the oxidation kinetics in $\mathrm{sCO}_{2}[2,3,24]$. The most comprehensive and long-term data (up to 10,000h) in $\mathrm{sCO}_{2}$ was generated at ORNL for the oxidation behavior of the three alloys $740 \mathrm{H}, 282$ and 625 [2-4]. An example of the recorded mass change data at $750{ }^{\circ} \mathrm{C}$ in atmospheric (1 bar) and high pressure (300 bar) $\mathrm{sCO}_{2}$ up to $10,000 \mathrm{~h}$ is shown in Figure 1.

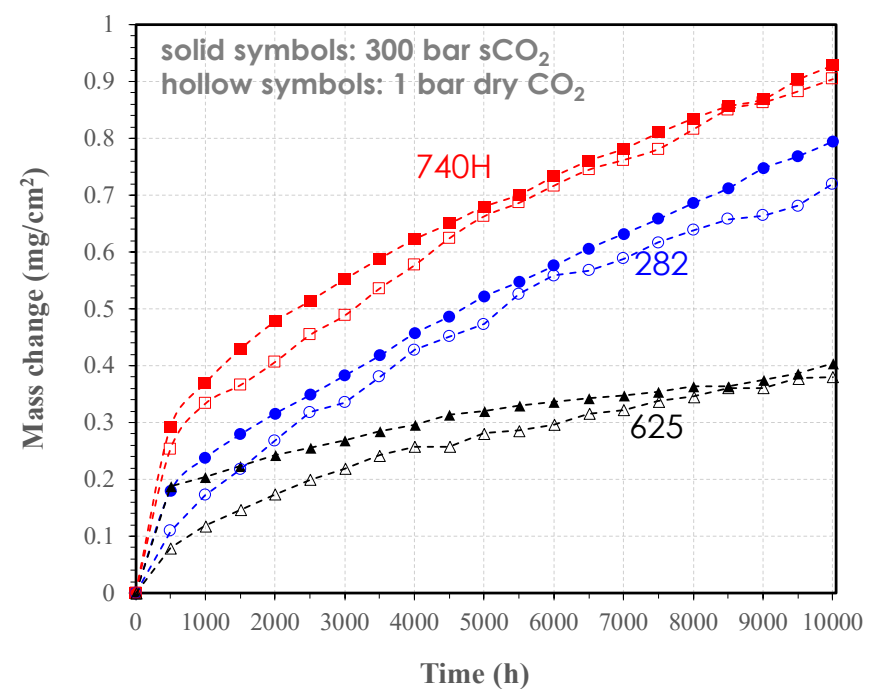

Figure 1: Measured mass change for $740 \mathrm{H}, 282$ and 625 during exposures in 1 bar and 300 bar $\mathrm{sCO}_{2}$ at $750^{\circ} \mathrm{C}$ [3]. 
Tucker et al. [15] summarized various data sources and reported mass change behavior of 625 and $740 \mathrm{H}$ in 200 bar $\mathrm{sCO}_{2}$ and this is summarized in Figure 2. Holcomb et al. [24] conducted tests in $\mathrm{sCO}_{2}$ at $730{ }^{\circ} \mathrm{C}$ and 207 bar and compared measured oxidation kinetics with values in the literature. Multiple reports and studies in the literature have shown that these alloys formed a protective external $\mathrm{Cr}_{2} \mathrm{O}_{3}$ layer during high temperature exposures in $\mathrm{sCO}_{2}$. Figure 3 shows the micrographs of the cross-sections of the three alloys at 700,750 and $800{ }^{\circ} \mathrm{C}$ after exposure for $5000 \mathrm{~h}$ in high pressure (300 bar) $\mathrm{sCO}_{2}$. An external $\mathrm{Cr}_{2} \mathrm{O}_{3}$ layer was observed on all three alloys with internal oxidation of $\mathrm{Al}$ and $\mathrm{Ti}$ in $740 \mathrm{H}$ and 282.

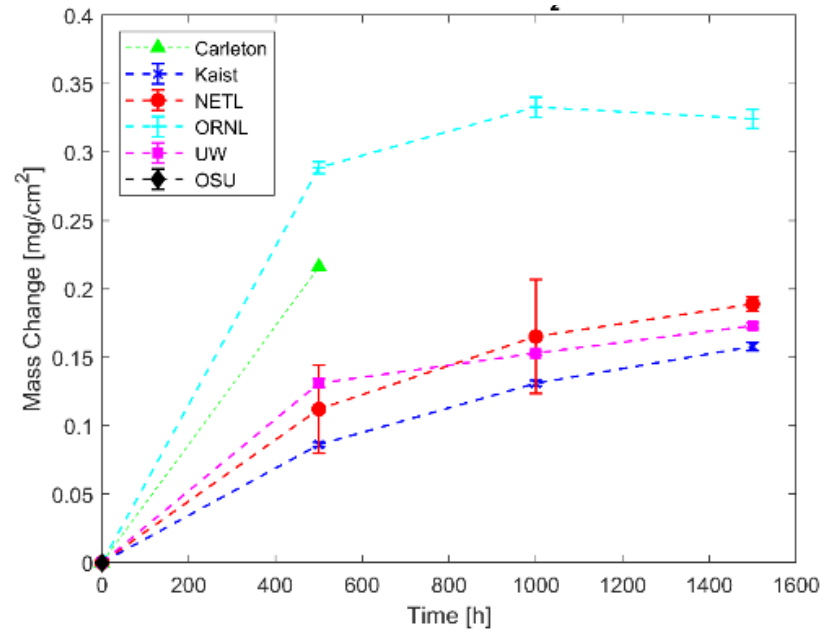

(a)

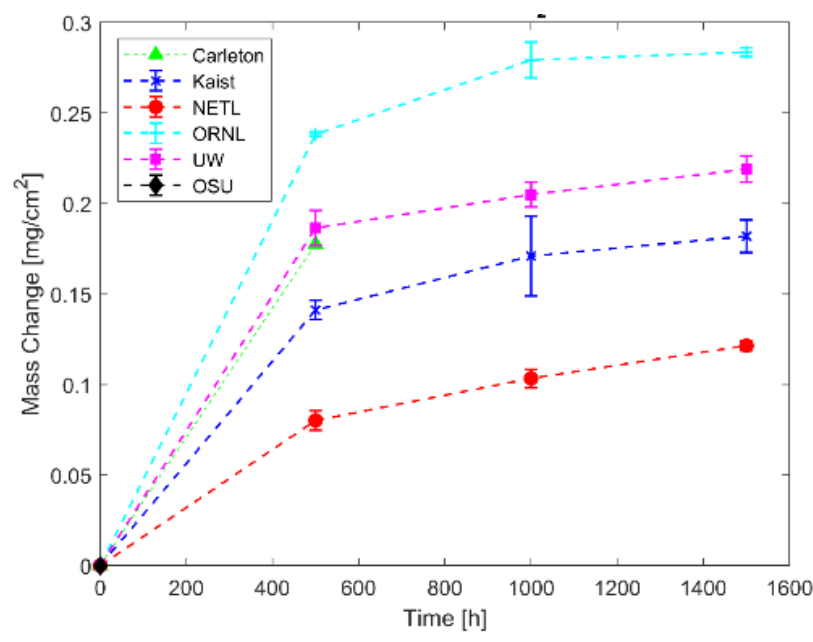

(b)

Figure 2: Measured mass change for (a) $740 \mathrm{H}$ and (b) 625 during exposures in 200 bar $\mathrm{sCO}_{2}$ at $700^{\circ} \mathrm{C}[15]$.

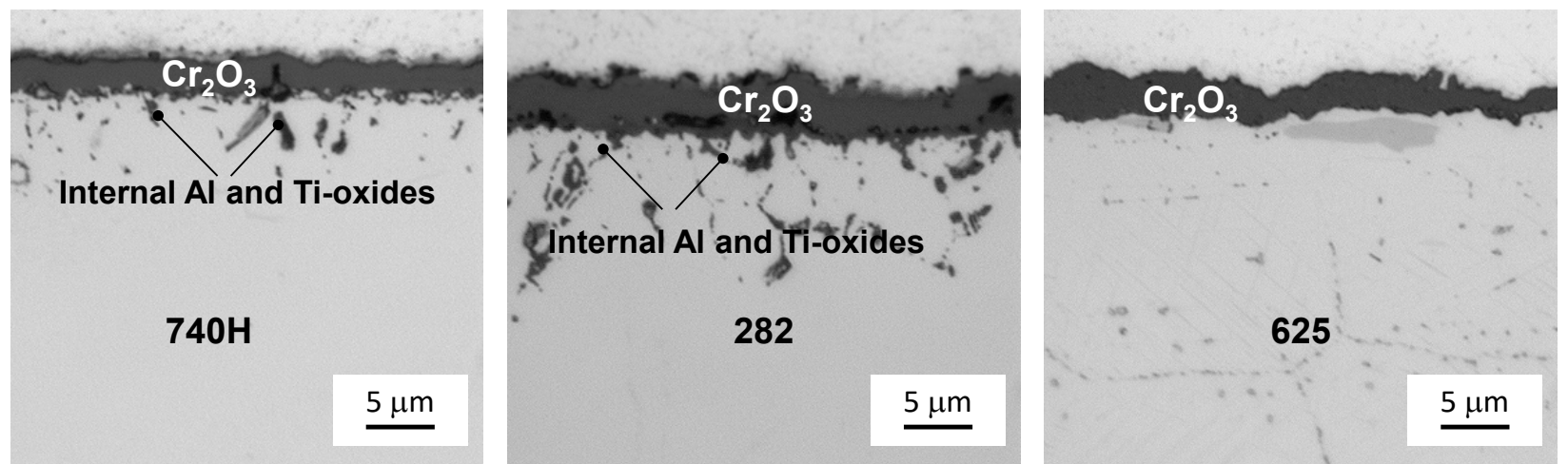

Figure 3: Light optical metallographic images of the cross-sections of $740 \mathrm{H}, 282$ and 625 after exposures for $5000 \mathrm{~h}$ at $750^{\circ} \mathrm{C}$.

The temperature range of the experiments conducted in the literature includes the maximum operating temperature of the chosen application as given in Table 2 . Oxidation rates $k_{p}$ of these alloys was calculated from recorded mass change data shown in Figure 1 [4] by fitting to a parabolic oxidation growth law, typically employed for growth of $\mathrm{Cr}_{2} \mathrm{O}_{3}$ oxide scales. Based on the gathered data, the corresponding metal loss rates $k_{\mathrm{c}}$ can then be calculated from these oxidation rates by using the alloy density and molar masses of oxygen and chromium. The Arrhenius plot of these calculated metal loss rates is given in Figure 4. Since pressure has been shown to have a negligible effect on the scaling kinetics of Ni-based alloys [4, 19, 24], test data gathered under different pressure conditions can be included to increase the statistical significance of experimental data. The data was used to estimate the loss of $\mathrm{Cr}$ in the alloy and the loss of sound metal (component thickness). 
It can be inferred from these studies and existing literature for $\mathrm{Ni}$-based alloys that an oxidation induced critical depletion of $\mathrm{Cr}$ in these alloys at the oxide-alloy interface will lead to non-protective oxidation behavior and faster scaling rates and metal loss. It was hence additionally concluded that gathering data on the compositional changes of $\mathrm{Cr}$ in these alloys as a function of time and temperature is essential for assessing the corrosion induced lifetime of the aforementioned alloys during high temperature exposures in $\mathrm{sCO}_{2}$. Access to specimens of $740 \mathrm{H}$, 282 and 625 exposed in $\mathrm{sCO}_{2}$ at ORNL enabled acquisition of EDS elemental concentration profiles.

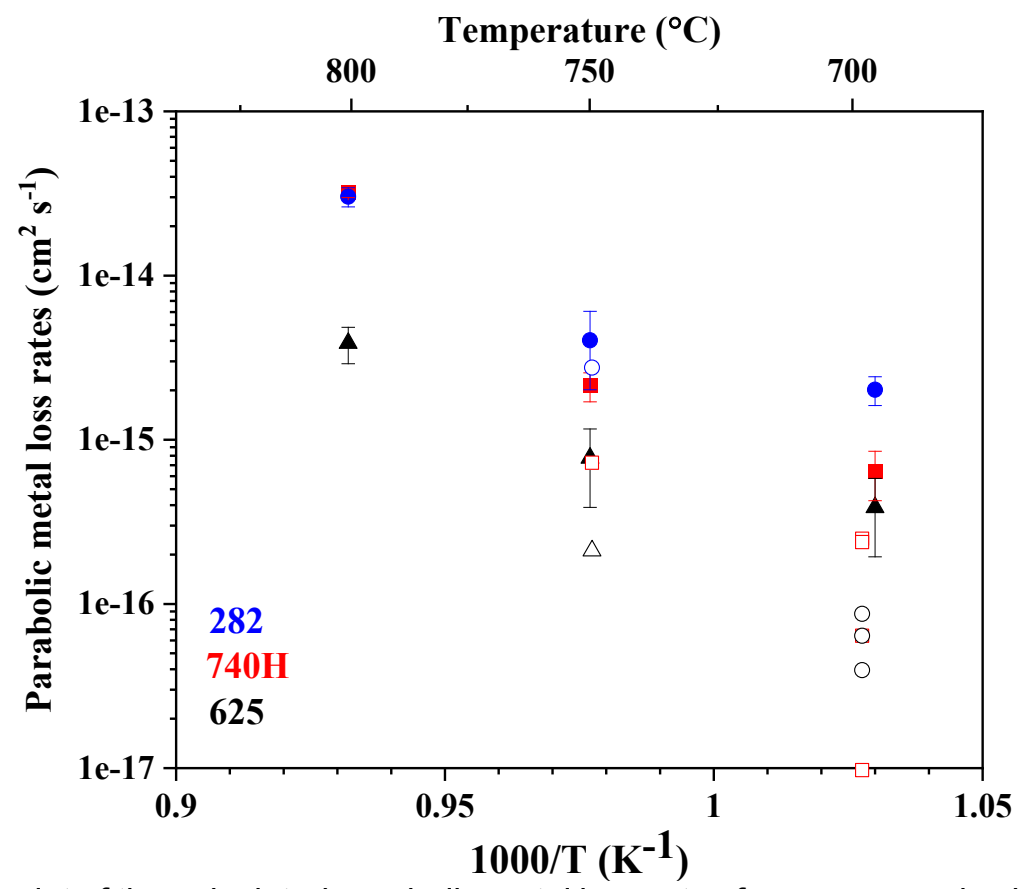

Figure 4: Arrhenius plot of the calculated parabolic metal loss rates from measured oxidation rates based on data in $[3,15,24]$. Solid symbols correspond to ORNL data while open symbols are data from literature.

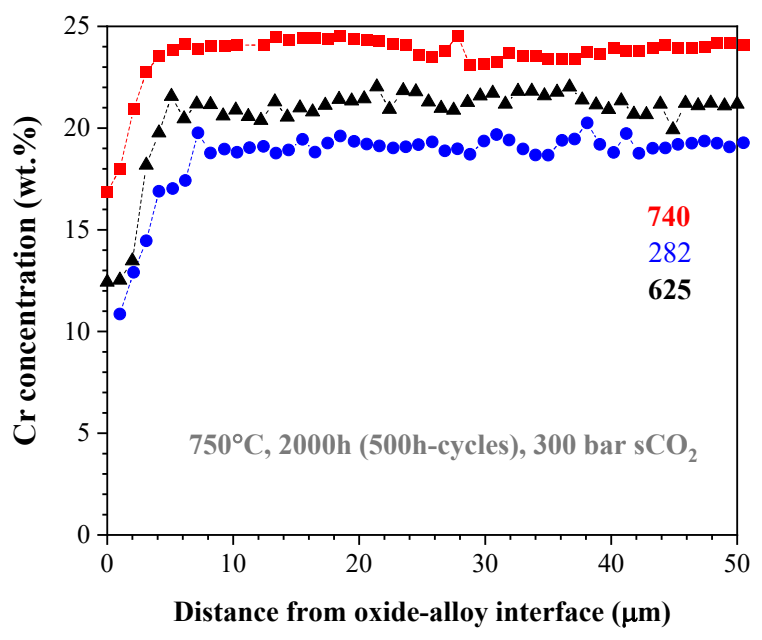

(a)

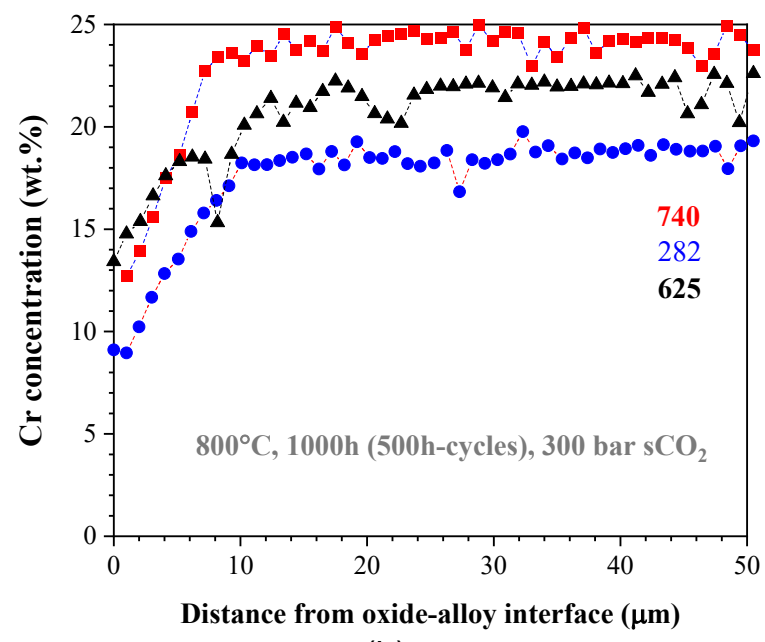

(b)

Figure 5: (a) Measured (EDS) $\mathrm{Cr}$ concentration profile in $740 \mathrm{H}, 282$ and 625 after exposure for (a) 2,000h (500h cycles) at $750^{\circ} \mathrm{C}$ and (b) $1,000 \mathrm{~h}\left(500 \mathrm{~h}\right.$ cycles) at $800^{\circ} \mathrm{C}$ in 300 bar $\mathrm{sCO}_{2}$. 


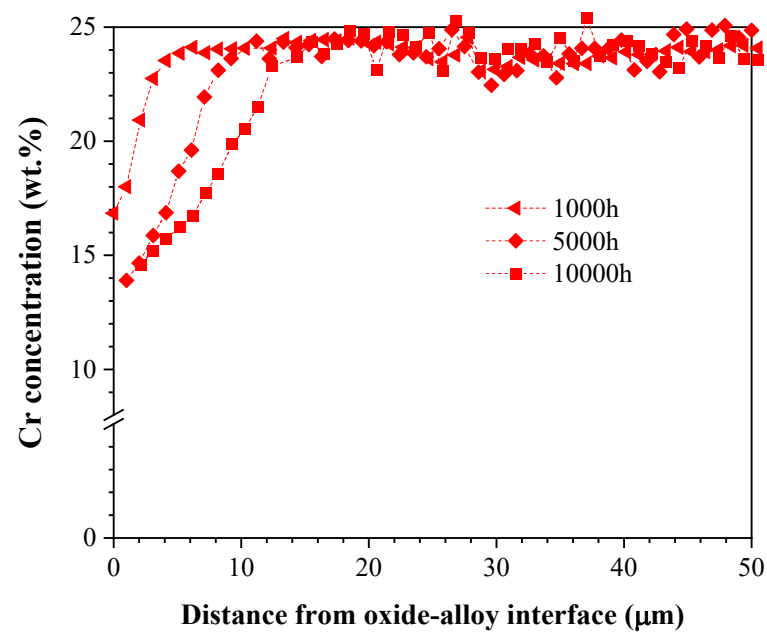

(a)

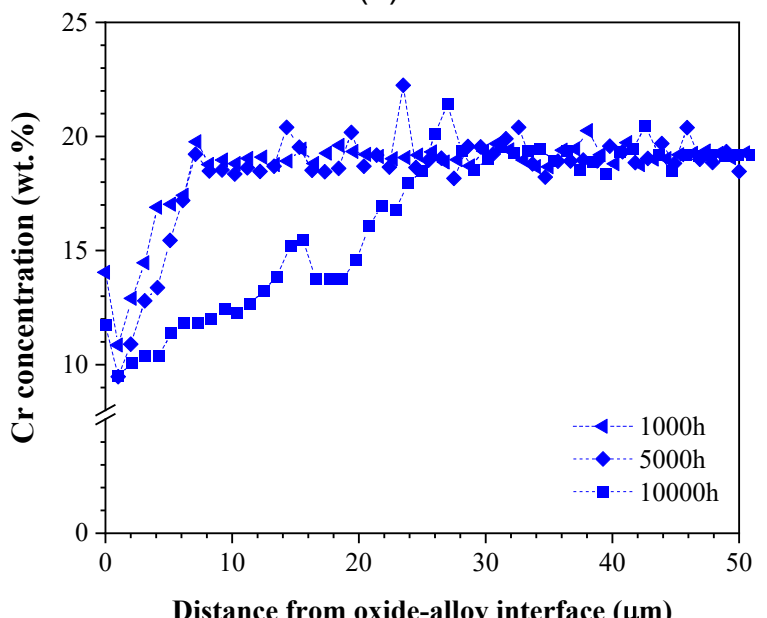

(b)

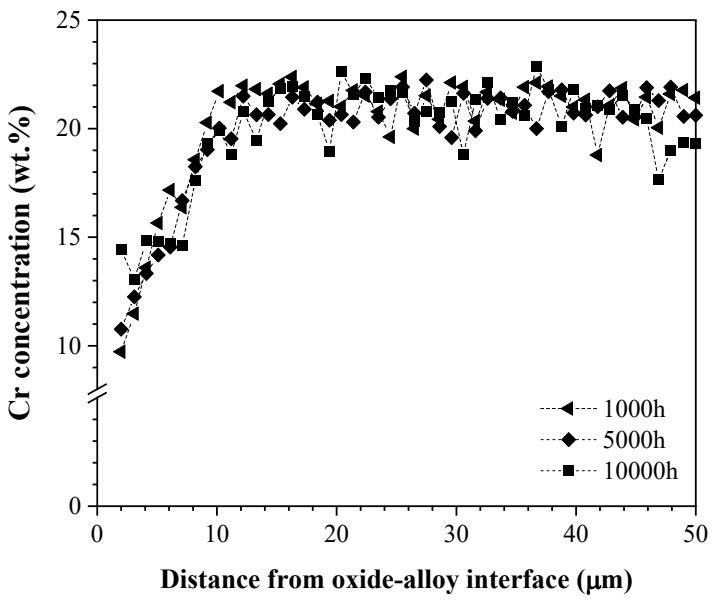

(c)

Figure 6: Time evolution of measured (EDS) Cr concentration profile in (a) $740 \mathrm{H}$, (b) 282 and (c) 625 after exposure for $1,000 \mathrm{~h}, 2000 \mathrm{~h}$ and $10,000 \mathrm{~h}\left(500 \mathrm{~h}\right.$ cycles) at $750^{\circ} \mathrm{C}$ in 300 bar $\mathrm{sCO}_{2}$.

Different time intervals were chosen to provide a temporal evolution of the $\mathrm{Cr}$ concentrations in the alloy. An example of the measured $\mathrm{Cr}$ concentration profile for $740 \mathrm{H}, 282$ 
and 625 after exposure for $2,000 \mathrm{~h}\left(500 \mathrm{~h}\right.$ cycles) at $750^{\circ} \mathrm{C}$ in 300 bar $\mathrm{sCO}_{2}$ is shown in Figure $5 \mathrm{a}$. Figure 6 shows the time evolution of measured $\mathrm{Cr}$ concentration profile in $740 \mathrm{H}, 282$ and 625 after exposure for $1,000 \mathrm{~h}, 5,000 \mathrm{~h}$ and $10,000 \mathrm{~h}$ (500h cycles) at $750{ }^{\circ} \mathrm{C}$ in 300 bar $\mathrm{sCO}_{2}$. Sparse measurements of $\mathrm{Cr}$ concentrations in the alloy are available in Wright et al. $\left(740 \mathrm{H}: 700{ }^{\circ} \mathrm{C}, 300 \mathrm{~h}\right.$ in commercial grade $\mathrm{CO}_{2}$ at 200 bar) and Tucker et al. $\left(625\right.$ and $740 \mathrm{H}: 700{ }^{\circ} \mathrm{C}, 1500 \mathrm{~h}$ in $\mathrm{CO}_{2}$ at 200 bar).

\section{Corrosion Data in molten chloride salts}

An extensive literature review was conducted to collect corrosion data for $740 \mathrm{H}, 282$ and 625 in molten chloride salts relevant to CSP applications such as $\mathrm{KCl}, \mathrm{MgCl}_{2}$ and $\mathrm{NaCl}$ between $700-800^{\circ} \mathrm{C}$. Although there have been studies reporting corrosion behavior of Ni-based alloys in molten chloride salts [31-35], to the best of our knowledge, there is almost no relevant corrosion data, in terms of measured concentration profiles and depths of attack for exposure of the aforementioned alloys in molten chloride salts. Furthermore, the lack of a standard procedure for testing corrosion in molten salts results in non-representative mass change measurements which complicates quantifying extent of corrosion damage. The main variables resulting in discrepancies of reported corrosion rates are a lack of consistency in salt chemistry (different purification procedures), use of different container materials (dissimilar capsule/test material combinations) and employing distinct test procedures (capsule/loop tests).

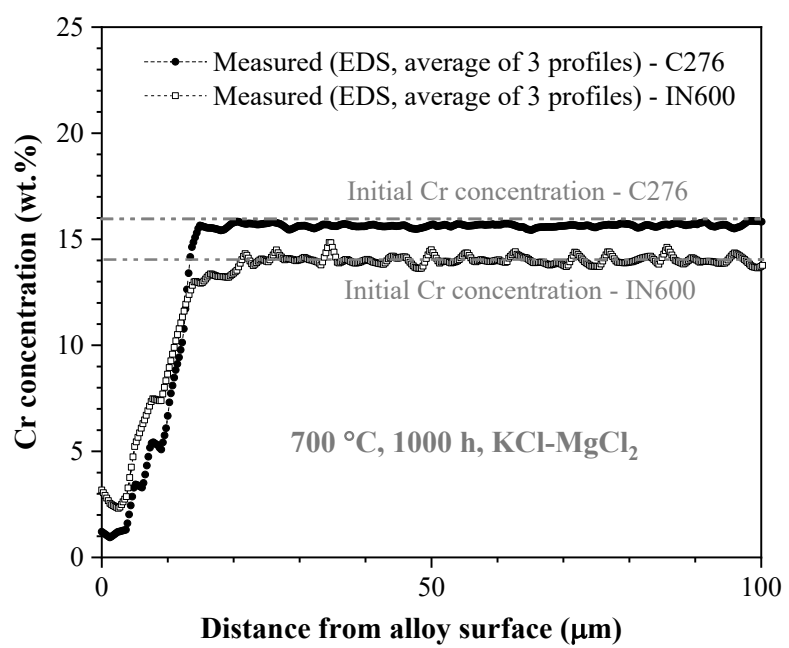

(a)

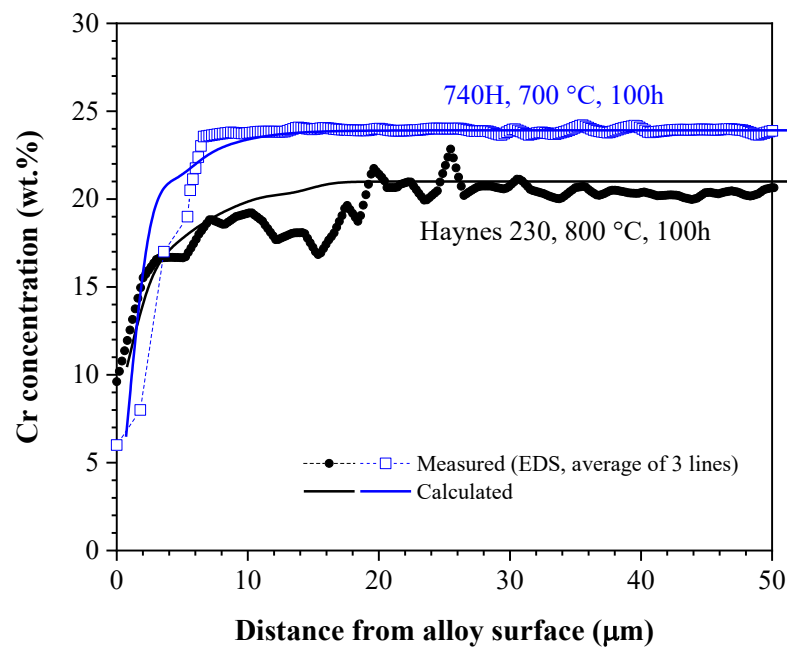

(b)

Figure 7: Measured (EDS) Cr concentration profile in (a) alloys 600 and C276 after exposure for 1000h in purified $\mathrm{KCl}-\mathrm{MgCl} 2740 \mathrm{H}, 282$ and 625 after exposure for (a) 2,000h (500h cycles) at $750^{\circ} \mathrm{C}$ and (b) $1,000 \mathrm{~h}\left(500 \mathrm{~h}\right.$ cycles) at $800^{\circ} \mathrm{C}$ in 300 bar $\mathrm{sCO}_{2}$.

Sun et al. [35] evaluated the corrosion behavior of seven Ni-based alloys in the ternary $\mathrm{NaCl}-\mathrm{KCl}-\mathrm{MgCl}_{2}$ under $\mathrm{N}_{2}$ atmosphere at $700{ }^{\circ} \mathrm{C}$ for $100 \mathrm{~h}$. Depths of attack ( $\mathrm{Cr}$ depletion) were reported but no concentration profiles were provided. The salt was however only oven-dried and residual moisture was suggested to have been the main cause of observed corrosion, although no clear evidence was given for this reasoning. Singh at al. placed alloy 625 specimens in graphite foam infiltrated with $\mathrm{MgCl}_{2}$ up to $1000 \mathrm{~h}$ at $750^{\circ} \mathrm{C}$. The set up was hermetically sealed in Incoloy $800 \mathrm{H}$ cylinders. The depth of attack and $\mathrm{Cr}$ depletion was measured after time intervals of 100, 200, 500 and 1000h. Although this limited data (e.g., one temperature) can be employed to validate the model, the alloys were exposed in graphite foams and the known reactivity of $\mathrm{Cr}$ with $\mathrm{C}$ can lead to different results compared to exposures of Ni-based alloys in purified molten 
chloride salts $[10,36]$. There is limited corrosion data generated at ORNL for $740 \mathrm{H}$ exposed in purified $\mathrm{KCl}-\mathrm{MgCl}_{2}$. Given the lack of data for the corrosion behavior of $740 \mathrm{H}, 282$ and 625, it was proposed to assimilate existing corrosion data for Ni-based alloys at temperatures of interest (700$800{ }^{\circ} \mathrm{C}$ ) in purified $\mathrm{KCl}-\mathrm{MgCl}_{2}$. For this purpose, additional data from previously conducted tests in purified $\mathrm{KCl}-\mathrm{MgCl}_{2}$ for alloys 230, 600 and $\mathrm{C} 276$ generated under the award number CPS 33873 "Progression to Compatibility Evaluations in Flowing Molten Salts" was employed for validation of the model predictions. Figure $13 a$ and $b$ show an example for this type of data.

\section{Creep Data}

Initially, the project focus was on demonstrating the correlation between Larson-Miller Parameter (LMP) and the strengthening phases in the alloys. However, based on the input provided by the industry, accumulated creep strains had been identified as a primary mode of failure for the heat exchanger components. An augmented effort was undertaken beyond the technical work plan to describe the complete creep behavior of the alloys to enable predictions of time to specific creep strains. All available Larson-Miller Parameter (LMP) data for $740 \mathrm{H}, 282$ and 625 to include the relevant operating conditions (Table 2) was assimilated. Additionally, all available creep data (creep strain curves) on the three alloys was gathered and corresponding metadata such as aging conditions and material geometry were recorded. For alloy 625 , only creep rupture data was available.

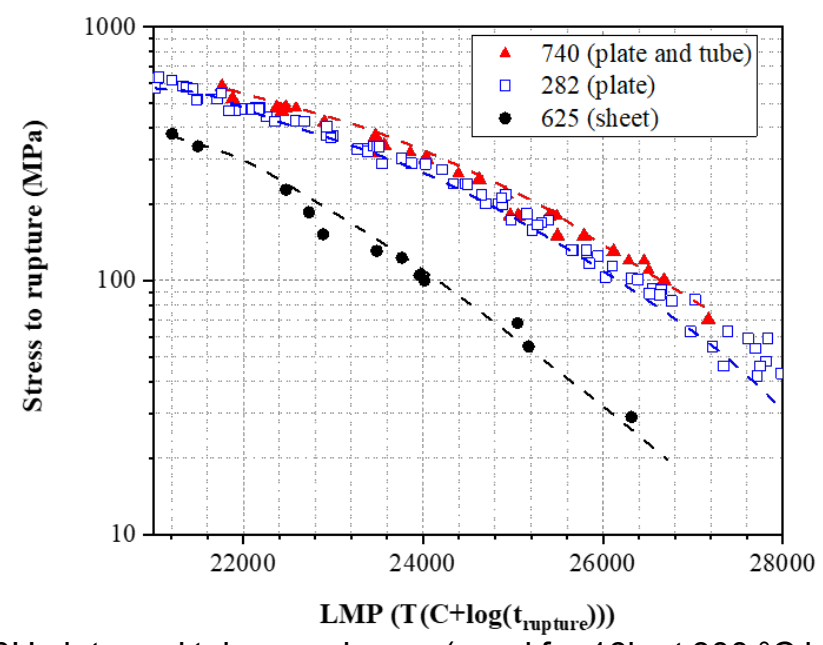

Figure 8: LMP plot for $740 \mathrm{H}$ plate and tube specimens (aged for $16 \mathrm{~h}$ at $800{ }^{\circ} \mathrm{C}$ in air) [37], 282 specimens (plate material) after aging for $4 \mathrm{~h}$ at $800^{\circ} \mathrm{C}$ in vacuum [30] and 625 sheet specimens (sheet material) in cold-rolled and annealed $\left(1052^{\circ} \mathrm{C}\right)$ condition for the Special Metals data, in as-received condition from ATI Allegheny Ludlum for ORNL data [38] and in solution annealed $\left(1100^{\circ} \mathrm{C}\right)$ condition for the ANL data [39].

A significant amount of creep rupture data for $740 \mathrm{H}$ has been generated within the framework of the A-USC materials development program between $700-850^{\circ} \mathrm{C}$ at stresses ranging from 50-500 MPa [27, 40, 41]. An example of the LMP data for plate and tube specimens aged for $16 \mathrm{~h}$ at $800^{\circ} \mathrm{C}$ in air is given in Figure 8. It is worth mentioning here that Render et al. [42] has evaluated the applicability of LMP and Wilshire approaches to predict the rupture times for the existing creep data for $740 \mathrm{H}$ from short-term data $(<5000 \mathrm{~h})$. Acceptable agreement has been reported using the Wilshire approach which provides the current project with a model to estimate the rupture times as a function of stress and temperature without the need to extract it from experimental data.

Pint et al. performed creep testing of 282 plate material at ORNL between 593-927 ${ }^{\circ} \mathrm{C}$ at stresses ranging from $20-800 \mathrm{MPa}$ with the goal of qualifying and deploying the 
alloy in advanced ultra-supercritical coal-fired boilers [30]. This data was provided for developing the intended model in the current project.

Existing creep data for 625 sheet specimens generated at ORNL [38] and at ANL [39] was assimilated and the corresponding LMP plot for the data is shown in Figure 8. Additionally, data from the alloy manufacturer Special Metals was also included resulting in a dataset encompassing temperatures between $593-1000^{\circ} \mathrm{C}$ and stresses from $30-600 \mathrm{MPa}$. There is generally a good agreement between all data sources. The LMP plot for $740 \mathrm{H}, 282$ and 625 is given in Figure 8. Figure 9 shows a few examples from the assimilated creep curves for $740 \mathrm{H}$ and 282 .

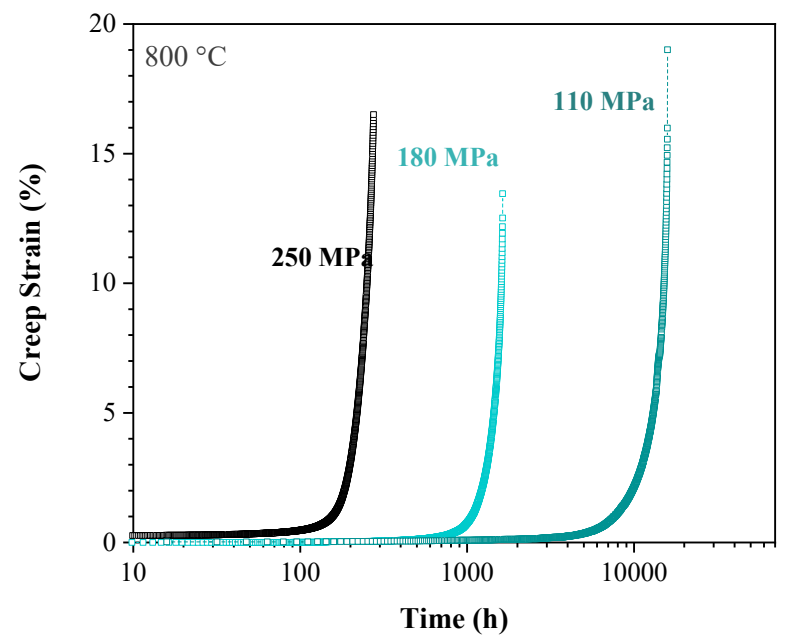

(a)

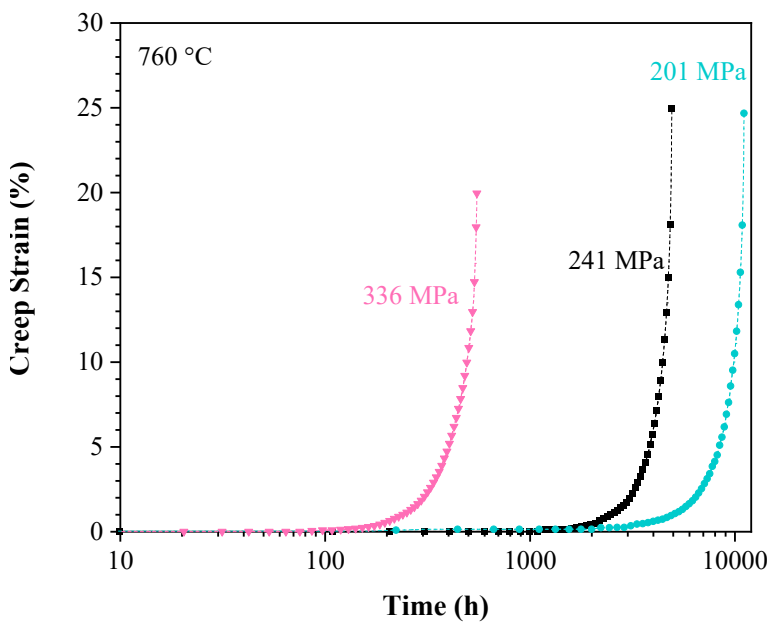

(b)

Figure 9: Measured creep strains for (a) $740 \mathrm{H}$ at $800{ }^{\circ} \mathrm{C}$ and (b) 282 at $760{ }^{\circ} \mathrm{C}$.

Microstructural characterization of the failed specimens after creep testing provided partial evidence to the failure mechanism. Figure 10 shows the light microscopy images along the edge of the gauge section after the creep rupture of 282 specimens at $816^{\circ} \mathrm{C}$ and three different rupture times. It can be inferred from the images that crack propagation is mainly observed in the $\gamma^{\prime}-$ depleted zone. The outer oxide was identified to be mainly $\mathrm{Cr}$-rich $\left(\mathrm{Cr}_{2} \mathrm{O}_{3}\right)$ with interspersed Tirich oxides. Internal oxidation of Al was additionally observed. Significant formation of the $\eta$-phase $\left(\mathrm{Ni}_{3} \mathrm{Ti}\right)$, seen as platelets in the $\gamma^{\prime}$-depleted zone, was identified.
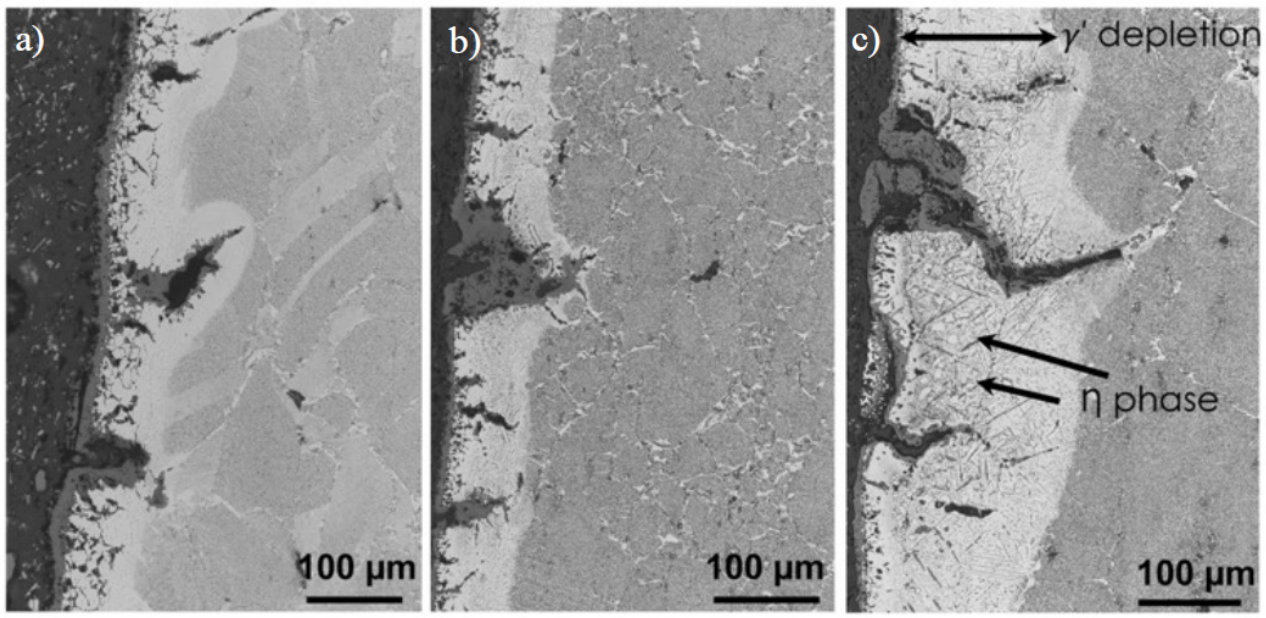

Figure 10: Light microscopy images showing microstructure along edge of the gauge section after the creep rupture of base metal 282 specimens at $816^{\circ} \mathrm{C}$ (a) $102 \mathrm{MPa}$ for $7911.7 \mathrm{~h}$, (b) $101 \mathrm{MPa}$ for 14182 $\mathrm{h}$ and (c) $100 \mathrm{MPa}$ for $16914 \mathrm{~h}$ [30]. 
In addition to the thermal aging processes, compositional changes occurring in the alloy subsurface during high temperature exposures in $\mathrm{sCO}_{2}$ can also drive dissolution of strengthening phases. This is shown for 282 and $740 \mathrm{H}$ in Figure 11. The denuded zone in the alloy subsurface is evident with complete depletion of $\gamma^{\prime}$-phase. The existing evidence supports the hypothesis that $\gamma^{\prime}$-depletion in the alloy subsurface, especially at the grain boundaries, driven by the oxidation induced loss of $\mathrm{Cr}$ (external scale) and $\mathrm{Al}$ (internal oxidation) is most likely one of the creep failure mechanisms in addition to the expected coarsening of the $\gamma^{\prime}$-phase. Supporting evidence for this hypothesis was given by Shingledecker et al. [40] for the creep rupture behavior of $740 \mathrm{H}$. The authors concluded based on microstructural evidence that the creep rupture strength and ductility of $740 \mathrm{H}$ was most likely governed by the formation of $\gamma^{\prime}$-denuded regions along the grain boundaries. Whether the formation of the $\eta$-phase $\left(\mathrm{Ni}_{3} \mathrm{Ti}\right)$ and the accompanying depletion of $\mathrm{Ti}$ further destabilizes the matrix needs to be ascertained.

With this knowledge, the depth of $\gamma^{\prime}$-depletion in the precipitation strengthened $740 \mathrm{H}$ and 282 alloys was measured with the help of micrographs (light optical microscopy and electron microscopy) from the specimens exposed at ORNL in $\mathrm{sCO}_{2}[3,4]$. As an example, Figure 12a shows the temporal evolution of the square root of the depth of $\gamma^{\prime}$-depletion measured in the $740 \mathrm{H}$ and 282 specimens after exposure in 300 bar $\mathrm{sCO}_{2}$. A linear correlation shows that the growth of the $\gamma^{\prime}$-depletion zone, $d$ follows the parabolic growth law $d=\left(g^{\star} t\right)^{1 / 2}$, where $g$ is a rate constant and $t$ is time, suggesting diffusion-controlled growth. The rate constant $g$ was estimated for $740 \mathrm{H}$ and 282 at 700,750 and $800{ }^{\circ} \mathrm{C}$ and a corresponding temperature dependence (Arrhenius law) was derived, as shown in Figure 12b.

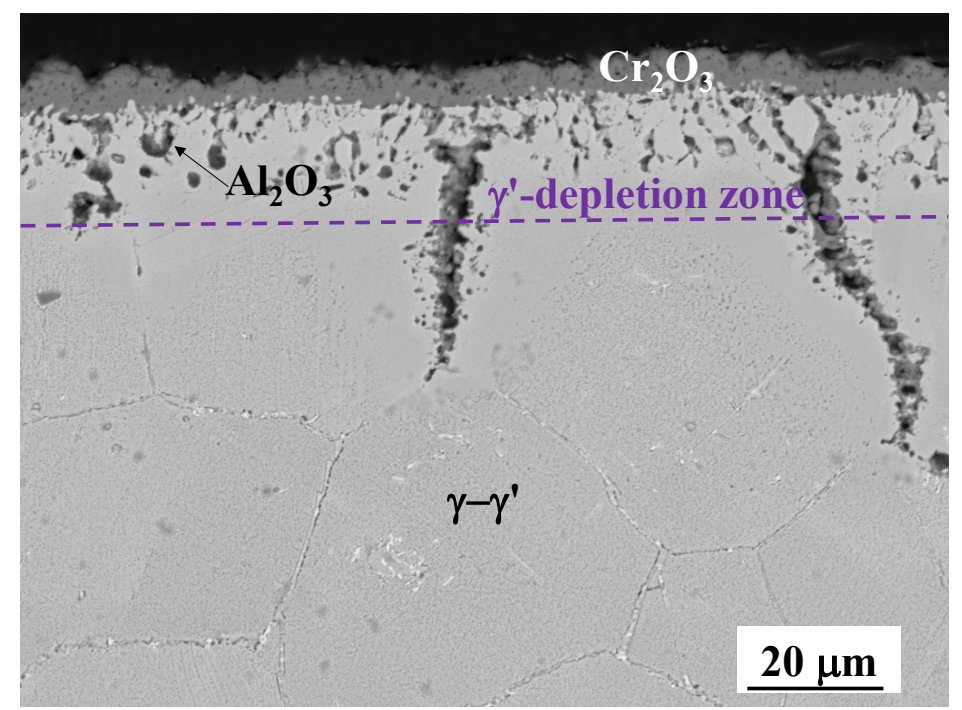

Figure 11: Corrosion induced degradation of the strengthening $\gamma^{\prime}$-phase in 282 after $11,000 \mathrm{~h}$ at $760{ }^{\circ} \mathrm{C}$ and $201 \mathrm{MPa}$.

625 is a solid solution strengthened material through additions of Mo and $\mathrm{Nb}$ but Cr-rich carbides and intermetallic phases such as $\gamma^{\prime \prime}$ and $\delta$ are known to precipitate during high temperature exposures and aid in increasing the creep rupture ductility $[43,44]$. The $\delta$-phase is the most thermodynamically stable phase in 625 up to $900^{\circ} \mathrm{C}$ along with $\mathrm{Cr}$ - and Mo-rich carbides that are stable well beyond $1000^{\circ} \mathrm{C}$. Both the $\delta$-phase and carbides are known to dissolve in the alloy subsurface during high temperature exposures in $\mathrm{SCO}_{2}$ and these are relevant microstructural changes to consider as influencing parameters in the creep behavior of 625 . The measured temporal evolution of the depth of $\delta$-phase dissolution at 700,750 and $800{ }^{\circ} \mathrm{C}$ after exposure in $\mathrm{sCO}_{2}$ after different time intervals was included in the dataset. 


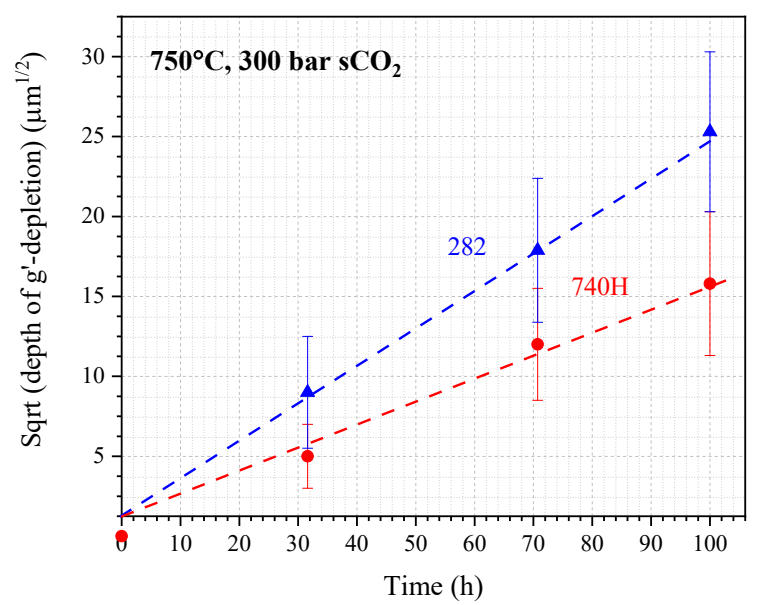

(a)

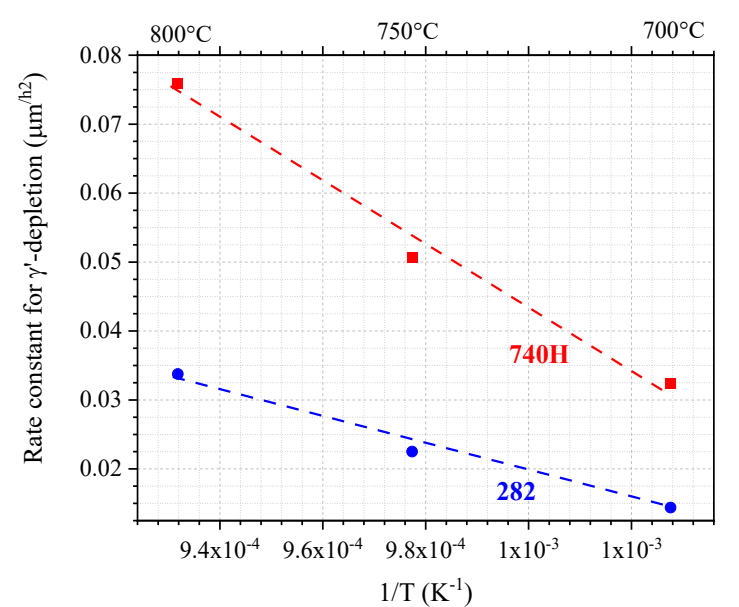

(b)

Figure 12: (a) Time dependence of $\gamma^{\prime}$-depletion and (b) temperature dependence of rate constant for $\gamma^{\prime}$ depletion in $740 \mathrm{H}$ and 282 at $750 \mathrm{C}$ in 300 bar $\mathrm{sCO}_{2}$.

Milestone 1.1.1: Establish basis for model development by defining application and application-specific operating conditions

The corrosion and creep data for $740 \mathrm{H}, 282$ and 625 discussed was organized in a master spreadsheet to enable easy access for the model development and validation. is thereby achieved. The assimilated database represents the operating conditions of the heat exchanger as tabulated in Table 2.

\subsubsection{Task 2: Model development}

\subsubsection{Subtask 2.1: Integrate models to describe physics for degradation of identified materials}

\section{Corrosion modeling in $\mathrm{sCO}_{2}$}

A protective chromia scale has been shown to form on the candidate alloys up to $15,000 \mathrm{~h}$ at $750^{\circ} \mathrm{C}$ [4]. However, it has been shown previously that a protective external chromia formation can most likely not be sustained below a critical value of $\mathrm{Cr}$ concentration at the scalelalloy (OIM) interface. The critical value was given to be $10 \mathrm{wt} \%[13,45]$ for chromia-forming Ni-based alloys. The concentration of $\mathrm{Cr}$ at the scalelalloy interface is governed by its consumption due to oxidation (oxidation kinetics) and its rate of transport from the alloy to the OIM interface (diffusion coefficient). For the case of parabolic oxidation kinetics, the $\mathrm{Cr}$ concentration at the interface is dependent on the initial $\mathrm{Cr}$ concentration and the ratio between the oxidation constant $k_{\mathrm{p}}$ and the diffusion coefficient of $\mathrm{Cr} D_{\mathrm{Cr}}$ in the alloy. In other words, if the oxide scale is not damaged due to spallation or depleted due to moisture induced evaporation of chromia, the interface $\mathrm{Cr}$ concentration remains constant [46]. This is supported by the experimental evidence in Figure 13 , where the $\mathrm{Cr}$ cocentration at the oxide-alloy interface is $\sim 14 \mathrm{wt} \%$ in $740 \mathrm{H}$ and 625 while it is just below 10 wt. \% in 282 after exposure for $10,000 \mathrm{~h}\left(500 \mathrm{~h}\right.$ cycles) at $750^{\circ} \mathrm{C}$ in 300 bar sCO $\mathrm{CO}_{2}$ Figure 14 shows the BSE images of the corresponding cross-sections. A predominantly $\mathrm{Cr}$-rich oxide scale $\left(\mathrm{Cr}_{2} \mathrm{O}_{3}\right)$ was observed on all three alloys. The presence of $\mathrm{Ti}$ in $740 \mathrm{H}$ and 282 results in formation of Ti-rich oxides in the external scale, either as clusters or as a layer at the gas/oxide interface. This was observed for both $740 \mathrm{H}$ and 282 . Ti has been reported to accelerate the 
oxidation kinetics of chromia-forming alloys and a critical depletion of $\mathrm{Cr}$ in the alloy will tend to result in the formation of more non-protective oxides (e.g., Ni, Co). The higher Ti content in 282 is most likely the reason for the deeper depletion of $\mathrm{Cr}$ driven by the low $\mathrm{Cr}$ concentration at the oxide/alloy interface. Consequently, the time to a critical $\mathrm{Cr}$ concentration has been implemented as a lifetime criteria.

\section{Corrosion modeling in molten salts}

Corrosion induced degradation in purified (negligible $\mathrm{O}_{2}$ and $\mathrm{H}_{2} \mathrm{O}$ contents) molten salts is distinct from the exposures in $\mathrm{sCO}_{2}$ since formation of an external $\mathrm{Cr}_{2} \mathrm{O}_{3}$ scale is not observed under these conditions. A number of molten salt corrosion mechanisms can be relevant during exposure of Fe and Ni-based alloys in molten halide salts [10].

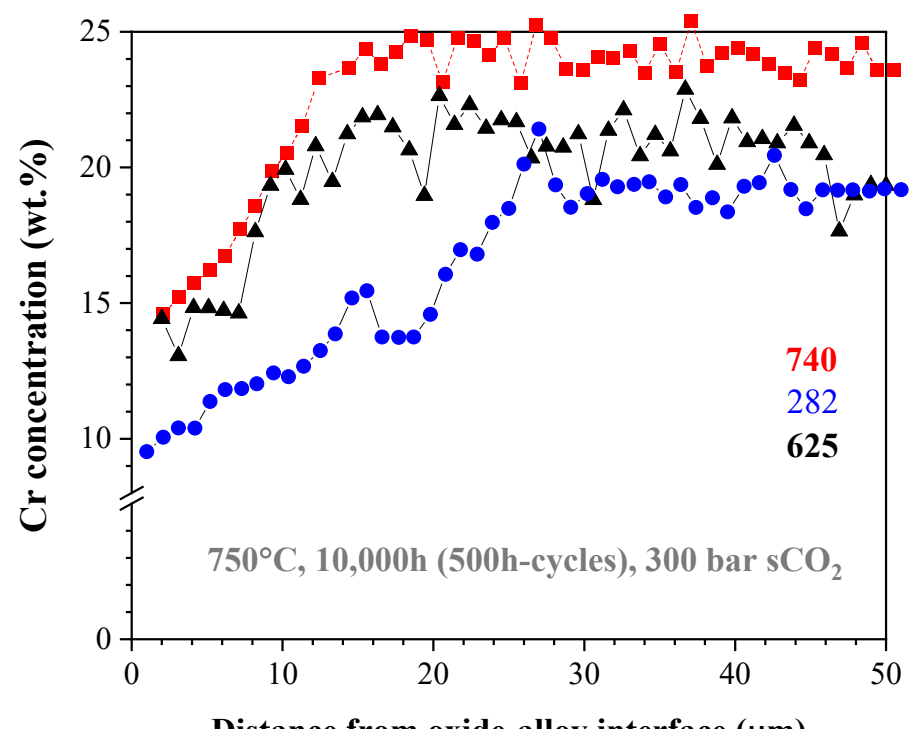

Distance from oxide-alloy interface $(\mu \mathrm{m})$

Figure 13: Measured (EDS) Cr concentration profile in 282 and (b) 625 after exposure for $1,000 \mathrm{~h}, 5,000 \mathrm{~h}$ and $10,000 \mathrm{~h}\left(500 \mathrm{~h}\right.$ cycles) at $750^{\circ} \mathrm{C}$ in 300 bar $\mathrm{sCO}_{2}$.
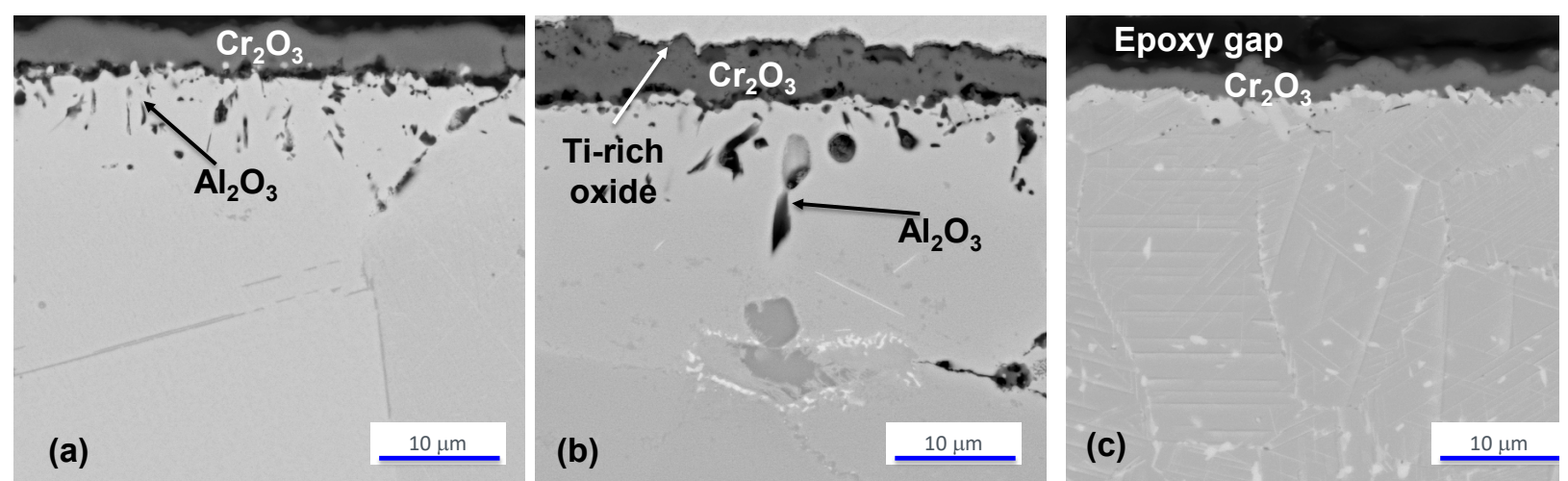

Figure 14: BSE images of the cross-sections of (a) $740 \mathrm{H}$, (b) 282 and (c) 625 after exposure for 10,000h (500h cycles) at $750^{\circ} \mathrm{C}$ in 300 bar $\mathrm{sCO}_{2}$.

Intrinsic corrosion driven by the difference in free energy of formation between the salt constituents and the most susceptible transition metal corrosion product is the most obvious mechanism. As is well known and often shown in the literature, halides of common alloying elements ( $\mathrm{Fe}, \mathrm{Ni}$ and $\mathrm{Cr}$ ) are less stable (less negative free energy of formation) than the halides 
that make up typical salt melts $\left(\mathrm{KCl}, \mathrm{MgCl}_{2}\right)$ [47]. Figure 15 shows the BSE image of the crosssection of alloy 230 after exposure for $92 \mathrm{~h}$ at $800{ }^{\circ} \mathrm{C}$ BSE image of the cross-section of alloy 230 after exposure for $92 \mathrm{~h}$ at $800{ }^{\circ} \mathrm{C}$ in Ni capsule in a Ni capsule. The measured $\mathrm{Cr}$ concentration profile is superimposed on the image to show the depletion of $\mathrm{Cr}$ in the corrosion-affected zone driven by its dissolution in the molten chloride salt. These compositional changes in the alloy subsurface can further drive dissolution of strengthening phases such as carbides as is evident in the case of alloy 230 (Figure 15). The width of such precipitate free zones usually corresponds to the depth of the corrosion-affected zone $[48,49]$. From a lifetime standpoint, predicting the depth of this corrosion-affected zone is relevant.

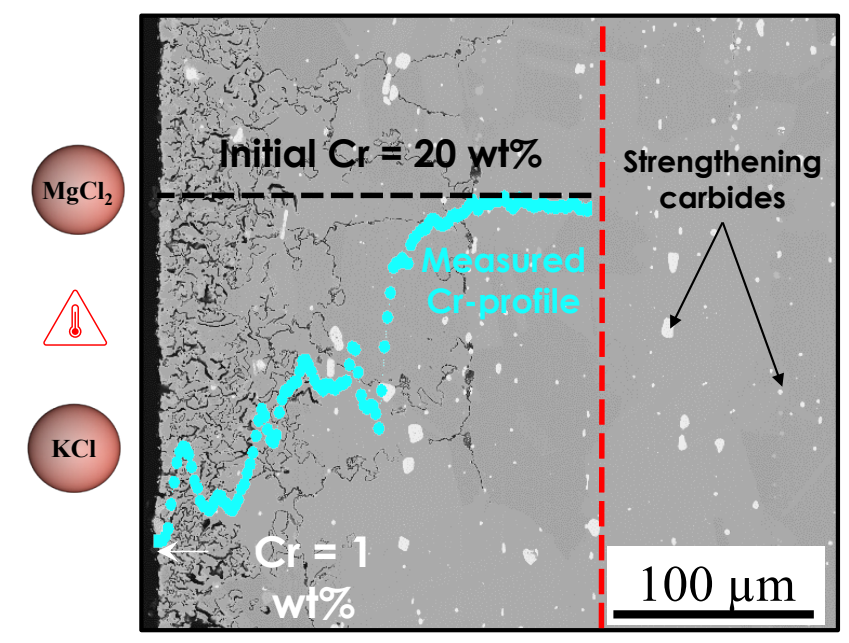

Figure 15: BSE image of the cross-section of alloy 230 after exposure for $92 \mathrm{~h}$ at $800{ }^{\circ} \mathrm{C}$ in purified commercial chloride salt [50] in a $\mathrm{Ni}$ capsule. The measured (EDS) $\mathrm{Cr}$ concentration profile is superimposed on the image.

\section{Modeling approach}

A two-pronged modeling approach was chosen to describe the corrosion induced compositional changes in the three alloys and predict corrosion-induced lifetimes. A previously developed physics-based coupled thermodynamic-kinetic model [51] modeling approach was employed to calculate the oxidation induced $\mathrm{Cr}$ depletion in the alloys during exposure in $\mathrm{sCO}_{2}$ and molten salt induced $\mathrm{Cr}$ dissolution during exposure in molten chloride salts. The procedure has been successfully employed to accurately predict the $\mathrm{Cr}$ depletion in 625 during exposure in $\mathrm{sCO}_{2}[4]$ and depths of attack in binary NiCr and commercial Ni-based alloys IN600 and C276 during exposure in purified molten chloride salts [10].

The modelling procedure utilizes commercially available thermodynamic data to calculate thermodynamic phase equilibria as a function of temperature and composition and kinetic data to determine the compositional evolution in the alloy. All chemical interactions between alloying elements are considered. The recession of the oxidelmetal (O\M) interface due to metal consumption to oxidation is calculated and the computational mesh is accordingly regenerated, thus enabling a more realistic calculation of e.g., $\mathrm{Cr}$ depletion profiles. The thermodynamic database TTNI8 and kinetic database MOBNI2 were employed in the calculations performed for the candidate alloys. A more detailed description of the modeling methodology can be found in [51]. In order to facilitate a simplified and rapid computation of $\mathrm{Cr}$-depletion profiles and lifetimes for the candidate alloys in the alloy selection tool, reduced order models were implemented. Furthermore, all potential users of the tool will not have access to the necessary thermodynamickinetic data required to employ the sophisticated model described earlier. The corrosion-induced 
$\mathrm{Cr}$ depletion will be calculated by numerically solving the following equation for the $\mathrm{Cr}$ concentration $C_{\mathrm{Cr}}$,

$$
\frac{\partial C_{C r}}{\partial t}=D_{C r} \frac{\partial^{2} C_{C r}}{\partial x^{2}}
$$

with the boundary conditions for the $\mathrm{Cr}$ flux $J_{\mathrm{Cr}}$,

$$
\begin{array}{ll}
J_{\mathrm{Cr}}=\frac{d}{d t}\left(k_{\mathrm{p}} t\right)^{n} & \text { at } x=0, t=t \\
J_{\mathrm{Cr}}=0 & \text { at } x=L, t=t
\end{array}
$$

Where $D_{\mathrm{Cr}}$ is the interdiffusion coefficient of $\mathrm{Cr}$ in the corresponding alloy, $k_{\mathrm{p}}$ is the temperature dependent corrosion rate constant (Figure 4) and $n$ is the exponent. Initial conditions are given by the initial chemical compositions of the alloys (Table 1) and average diffusion coefficients for $\mathrm{Cr}$ in $740 \mathrm{H}, 282$ and 625 were employed. The composition and temperature dependent average diffusion coefficients were estimated from available CALPHAD-based kinetic data [52]. The reduced order model provided $\mathrm{Cr}$ concentration profiles and depths of attack as a function of time and temperature. The correlation between compositional changes and dissolution of strengthening phases were estimated with the sophisticated coupled thermodynamic-kinetic model. These correlations were developed into simplified functions in the reduced order model. These functions also serve as input to the physics-based creep model.

\section{Numerical parameters}

The oxidation kinetics derived from the generated database discussed under Task 1 were implemented as a boundary condition on the alloy surface to simulate the oxidation-induced $\mathrm{Cr}$ loss for exposures in $\mathrm{sCO}_{2}$. This is an input property in the model. To account for potential spallation of the chromia scales after longer times or due to thermal cycling induced by abrupt shut-down procedures, $\mathrm{Cr}$ loss can be accordingly increased, thereby providing a parameter to the user to evaluate the influence on corrosion-induced lifetime

For exposures in molten $\mathrm{KCl}-\mathrm{MgCl}_{2}$ salts, it is hypothesized, based on the work conducted by DeVan [5], that if the dissolution rate of pure $\mathrm{Cr}$ in a particular salt is known, then the $\mathrm{Cr}$ dissolution rate in a multicomponent alloy can be scaled to the ratio of the Cr chemical activity since the Cr activity of pure $\mathrm{Cr}$ is one. The $\mathrm{Cr}$ dissolution rate in $740 \mathrm{H}, 282$ and 625 was estimated from previously conducted experiments at ORNL for dissolution of pure $\mathrm{Cr}$ in purified $\mathrm{KCl}-\mathrm{MgCl}_{2}$. Pure $\mathrm{Cr}$ specimens were exposed in purified $\mathrm{KCl}-\mathrm{MgCl}_{2}$ at $700{ }^{\circ} \mathrm{C}$ and $800{ }^{\circ} \mathrm{C}$ for up to $5000 \mathrm{~h}$ and the $\mathrm{Cr}$ content in the salt was measured with ICP-OES (inductively coupled plasma-optical emission spectrometry) post-exposure at pre-defined intervals of 1000, 2500 and 5000h. A temperature dependent $\mathrm{Cr}$ dissolution rate was derived from the measured $\mathrm{Cr}$ content and the $\mathrm{Cr}$ loss kinetics were fitted to a power time law of the type $(k t)^{n}$ where $k$ is the temperature dependent corrosion rate constant and $n$ is the exponent. The temperature and composition dependent chemical activity of $\mathrm{Cr}$ in $740 \mathrm{H}, 282$ and 625 was calculated from the thermodynamic database TCNI8 [53]. An outward Cr flux corresponding to the Cr loss estimated from the Cr dissolution rate of pure $\mathrm{Cr}$ and the chemical activity of the alloy was employed as a boundary condition at the alloy surface to simulate the corrosion process. Such an approach has been successfully employed to predict $\mathrm{Cr}$ depletion profiles and depths of attack for model and commercial Ni-based alloys [54]. The assumption of symmetry at the center of the sample provided the second spatial boundary condition of zero flux for all elements on the other side of the domain. The measured compositions of the alloy in the as-received state were used as the initial composition at time $t=0$. The model provides average element concentration profiles and depths of attack as a function of time and temperature. 


\section{Creep modeling}

To predict the lifetime based on accumulated creep strains, a microstructure-based statevariable (or damage-mechanics) approach for improved design, based on a previously proposed procedure by Dyson and Mclean [55-57], was adopted to describe the creep behavior of the three alloys. The method integrates contribution to creep damage by relevant degradation mechanisms (e.g., cavitation/cracking, particle coarsening, phase changes, dislocation accumulation)) allowing a material-specific evaluation of creep behavior). This is an advantage to empirical representations of creep behavior since an effective extrapolation to longer lives and more complex loading conditions requires that the differing mechanisms be integrated in the creep equations. Furthermore, this allows integration of corrosion-induced damage, e.g., depletion of the strengthening phase which can be critical for thin components. A general description of the generic set of creep equations considering the relevant damage mechanism is given in Table 5 .

Table 5: Different damage mechanisms and corresponding rate equations

\begin{tabular}{|c|c|}
\hline Equation & Damage mechanism \\
\hline$\dot{H}=\frac{h^{\prime}}{\sigma}\left(1-\frac{H}{H^{*}}\right) \dot{\varepsilon}$ & Strain hardening and recovery \\
\hline$\dot{D_{\mathrm{d}}}=C_{4}\left(1-D_{\mathrm{d}}^{2}\right) \dot{\varepsilon}$ & Dislocation multiplication \\
\hline$\dot{D_{\mathrm{p}}}=\frac{K_{p}}{3}\left(1-D_{\mathrm{p}}\right)^{4}$ & Particle coarsening \\
\hline$\dot{D}_{\mathrm{sp}}=\frac{K_{\mathrm{sp}}}{d^{2} D_{\mathrm{sp}}}$ & Depletion of strengthening phases \\
\hline$\dot{\sigma}=\sigma \dot{\varepsilon}$ & Stress change during constant load test; necking \\
\hline
\end{tabular}

The overall creep strain rate $\dot{\boldsymbol{\varepsilon}}$ at a given stress $\sigma$ is given by,

$$
\dot{\varepsilon}=\frac{\dot{\varepsilon_{0}}}{1-D_{d}} \sinh \left[\frac{\sigma(1-H)}{\sigma_{0}\left(1-D_{p}\right)\left(1-D_{s p}\right)}\right]
$$

The dislocation density is expected to increase with continuing accumulation of creep damage. Multiplication of these dislocations has been proposed to be a key degradation mechanism contributing to tertiary creep in Ni-based superalloys [55, 58]. Particle coarsening is a relevant damage mechanism in precipitate strengthened alloys and applies to alloys $740 \mathrm{H}$ and 282, which are $y^{\prime}$-strengthened. Crack propagation due to growth and nucleation of cavities at grain boundaries accelerates creep damage and can be a critical damage mechanism in the tertiary creep regime. In addition to the typically included damage mechanisms, dissolution of the strengthening phase has also been included here to account for the corrosion-induced dissolution of strengthening precipitate phases in the alloys chosen here. This might be a critical damage mechanism and be life-limiting for thin components $(<0.5 \mathrm{~mm})$, with the depth of precipitatedenuded zones reaching up to $50 \%$ of the foil thickness resulting in significant reduction in creep strength. The symbols are defined in Table 6 . The volume fraction of the strengthening phase $\varphi_{\gamma^{\prime}}$ in $740 \mathrm{H}$ and 282 determined the parameters for strain hardening/recovery $H$ and the intrinsic strain rate $\dot{\varepsilon_{0}}$. The intrinsic strain rate is described as [58] ,

$$
\dot{\varepsilon}_{0}=C_{e p s o} * 2 \sqrt{\varphi_{\gamma^{\prime}}} *\left(1-\varphi_{\gamma^{\prime}}\right) *\left(\sqrt{\frac{\pi}{4}}-\sqrt{\varphi_{\gamma^{\prime}}}\right) * e^{\left(\frac{-Q}{R T}\right)}
$$


Table 6: Definition of symbols given in Table 5 and Eq. 4.

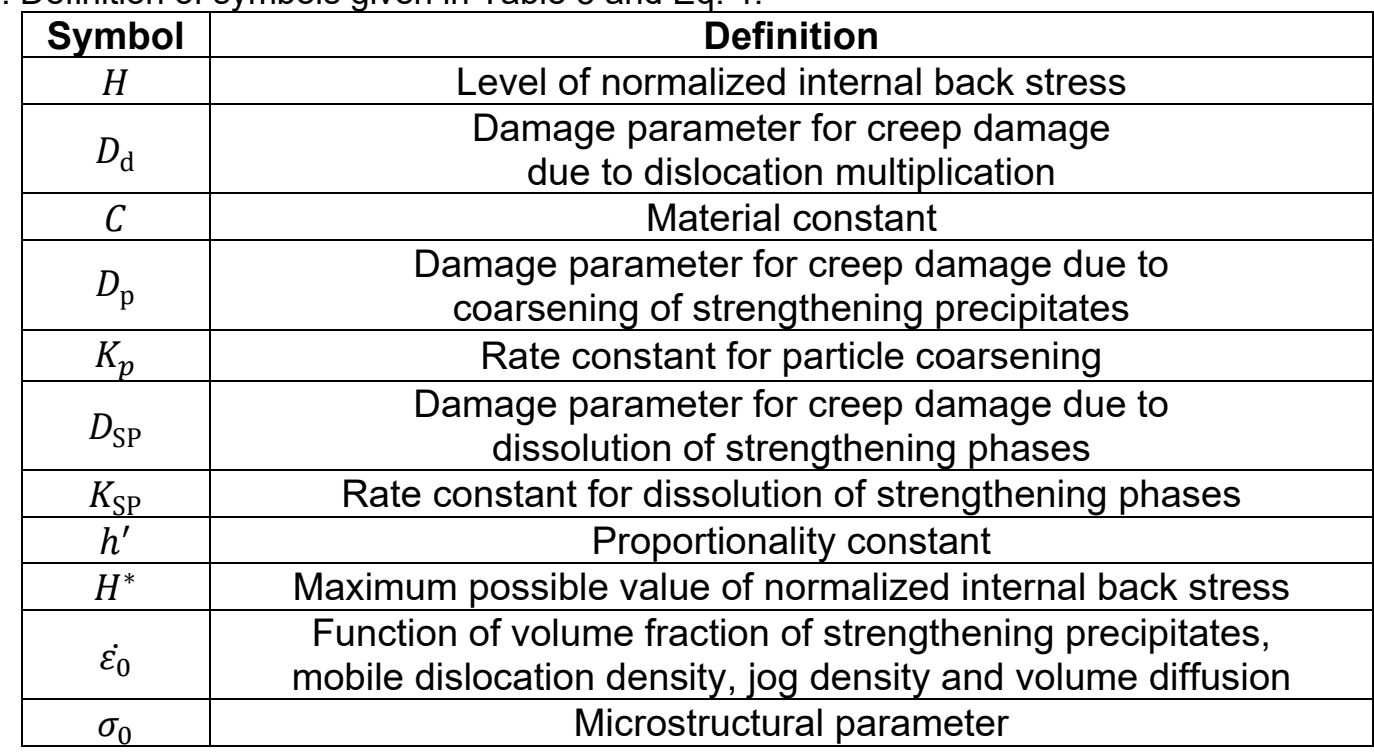

where $Q$ is the activation energy for volume diffusion (typically of the order of $300 \mathrm{~kJ} / \mathrm{mol}$ ) The temperature dependence of $\varphi_{\gamma^{\prime}}$ was derived from the available thermodynamic data [53] as follows,

$$
\varphi_{\gamma^{\prime}}=a_{S} * T^{3}+b_{S} * T^{2}+c_{S} * T+d_{S}
$$

The parameter $H^{*}$ is estimated as $2 * \varphi_{\gamma^{\prime}} /\left(1+\varphi_{\gamma^{\prime}}\right)$ while the microstructural parameter $\sigma_{0}$ is given as,

$$
\sigma_{0}=C_{1} *\left(1-\exp \left(\left(\frac{-C_{2}}{R T_{S}}\right)\left(\frac{T_{S}}{T}-1\right)\right)\right)
$$

with $C_{1}$ and $C_{2}$ are material constants and $T_{s}$ is solvus temperature of in $740 \mathrm{H}$ and 282 . The influence of mobile dislocation density and jog density, defined as $C_{\mathrm{eps} 0}$ on $\dot{\varepsilon}_{0}$ was estimated by fitting the solution of Eq. 4 to experimental data. The stress and temperature dependence of $C_{\mathrm{eps} 0}$ was described based on the formulations proposed by Pillai [59] for carbide strengthened Nibased alloys:

$$
C_{\text {eps } 0}=e^{\left(a+b * \ln \left(\frac{\sigma}{\sigma_{0.2}}\right)+c * \sinh \left(\frac{\sigma}{T}\right)+d * \ln \left(\frac{1}{T}\right)+f *\left(\frac{1}{T}\right)\right)}
$$

Eq. 8

To estimate the rate constant for particle coarsening, existing literature data for coarsening of $Y^{\prime}$ in $740 \mathrm{H}$ and 282 was employed [60-62]. The temperature dependent rate constant for the dissolution of strengthening phases, mainly relevant for $740 \mathrm{H}$ and 282 , was estimated from the data shown in Figure 12. The system of differential equations given by the rate equations in Table 5 and was solved and the solution was fitted to a subset of the measured creep data for 282 [30] and $740 \mathrm{H}[41,63]$ using the global minimization genetic algorithm procedure in MATLAB. About $40 \%$ of the data was used to estimate the model parameters $C_{1}, C_{2}, C_{\text {eps } 0}$ and $C_{4} .$. The available creep data for 625 was deemed insufficient to reliably estimate the model parameters. The stress dependence of the creep rupture data (LMP) for the three alloys was described as, 


$$
a_{r}+b_{r} \log (\sigma)+c_{r} \log (\sigma)^{2}+d_{r} \log (\sigma)^{3}
$$

All material dependent model parameters are saved in text files (accessible on the GITHUB platform) and are accordingly read by the creep model during simulations.

\subsubsection{Subtask 2.2: Perform test calculations to verify functionality of the model}

The modeling procedure described earlier was employed to perform a few test calculations to verify the functionality of the adopted methods. The corrosion induced $\mathrm{Cr}$ depletion was calculated for two cases: (i) in 625 for exposures in $\mathrm{sCO}_{2}$ and (ii) in $740 \mathrm{H}$ for exposures in molten $\mathrm{KCl}-\mathrm{MgCl}_{2}$ mixture. The creep behavior of 282 was modeled with the continuum damage mechanics approach described earlier.

\section{Predicting corrosion induced $\mathrm{Cr}$ depletion}

Figure 16a compares the measured (EDS) and calculated $\mathrm{Cr}$ concentration profile in 625 after exposure in $\mathrm{sCO}_{2}$ for $10,000 \mathrm{~h}$ at $750^{\circ} \mathrm{C}$. The model predicted an OM interface $\mathrm{Cr}$ concentration of $14.6 \mathrm{wt}$ \% while the measured concentration was about $14 \mathrm{wt} . \%$. There is good agreement between the measured and calculated $\mathrm{Cr}$ concentrations and depth of $\mathrm{Cr}$ depletion. Figure 16b compares the measured and calculated $\mathrm{Cr}$ concentration profiles in $740 \mathrm{H}$ after exposure in purified molten $\mathrm{KCl}-\mathrm{MgCl}_{2}$ for $100 \mathrm{~h}$ at $700^{\circ} \mathrm{C}$. The predicted depth of $\mathrm{Cr}$ depletion (depth of corrosive attack) agrees well with experimental measurements.

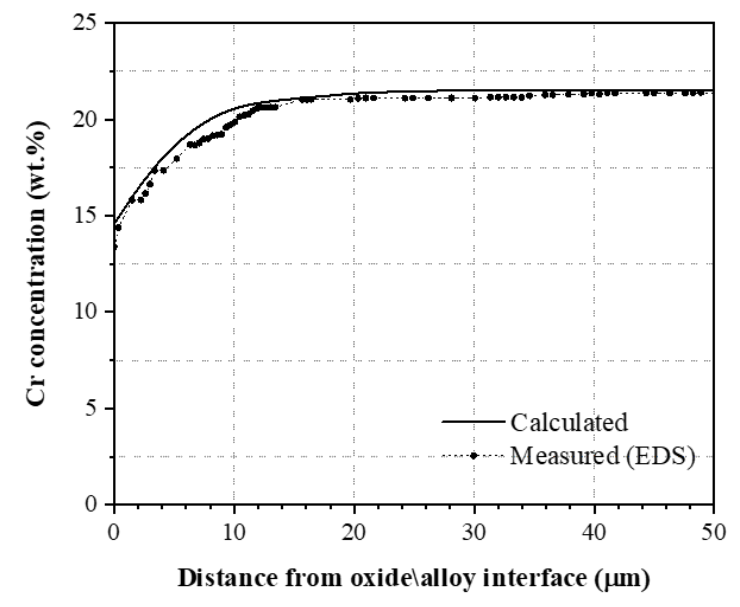

(a)

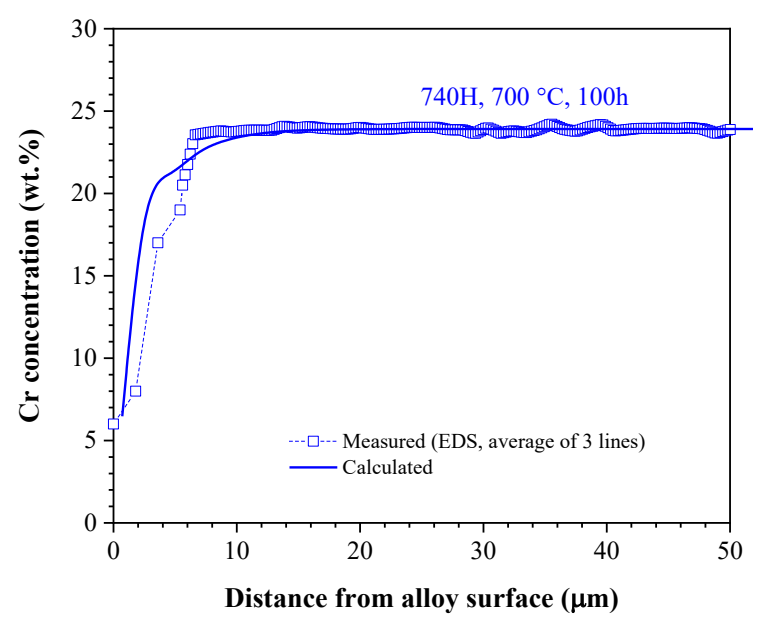

(b)

Figure 16: (a) Measured (EDS) and calculated $\mathrm{Cr}$ concentration profile in 625 after exposure for 10,000h (500h cycles) at $750^{\circ} \mathrm{C}$ in 300 bar $\mathrm{sCO}_{2}$ and (b) in $740 \mathrm{H}$ after exposure for $100 \mathrm{~h}$ at $700^{\circ} \mathrm{C}$ in purified eutectic $\mathrm{KCl}-\mathrm{MgCl}_{2}$ mixture.

\section{Predicting creep behavior of 282 and $740 \mathrm{H}$}

As described under Subtask 2.1, solution given by Eq. 1 was fitted to a subset of the measured creep data for $740 \mathrm{H}$ and 282 . About $40 \%$ of the data was used to estimate the model parameters $\dot{\varepsilon}_{0}, \sigma_{0}, C, h^{\prime}$ and $H^{*}$. Figure 17a shows the comparison between the measured and calculated (fit) creep strains at two different conditions. The CDM model was able to predict the creep strains to an acceptable accuracy. Figure 17b shows the predicted strains for two test conditions not used in the fitting procedure. Figure 18a and $b$ compare the measured and calculated creep strains for $740 \mathrm{H}$ for three stresses at $750{ }^{\circ} \mathrm{C}$ and $800{ }^{\circ} \mathrm{C}$. Since the time to $2 \%$ 
creep strain is a lifetime criteria (feedback provided by Brayton), the predictions were within $500 \mathrm{~h}$ at all conditions for $740 \mathrm{H}$ and 282.

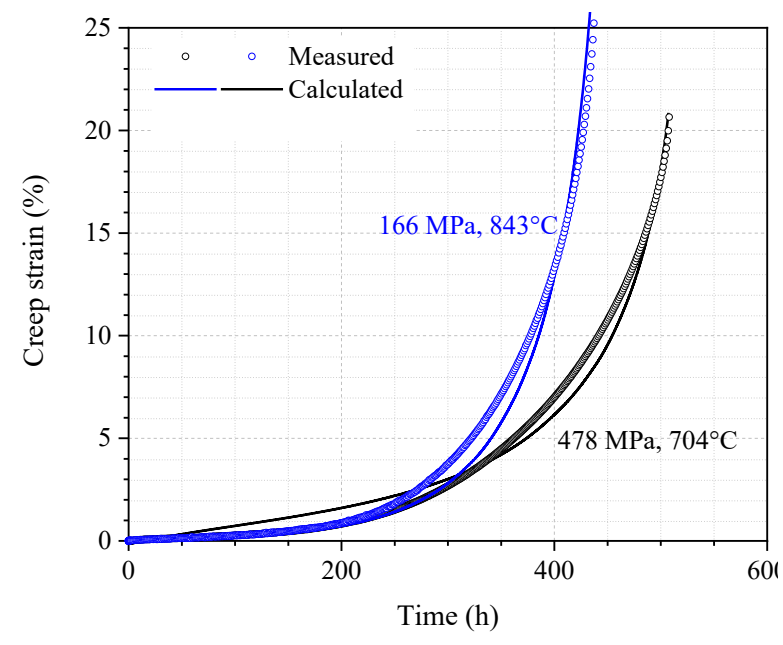

(a)

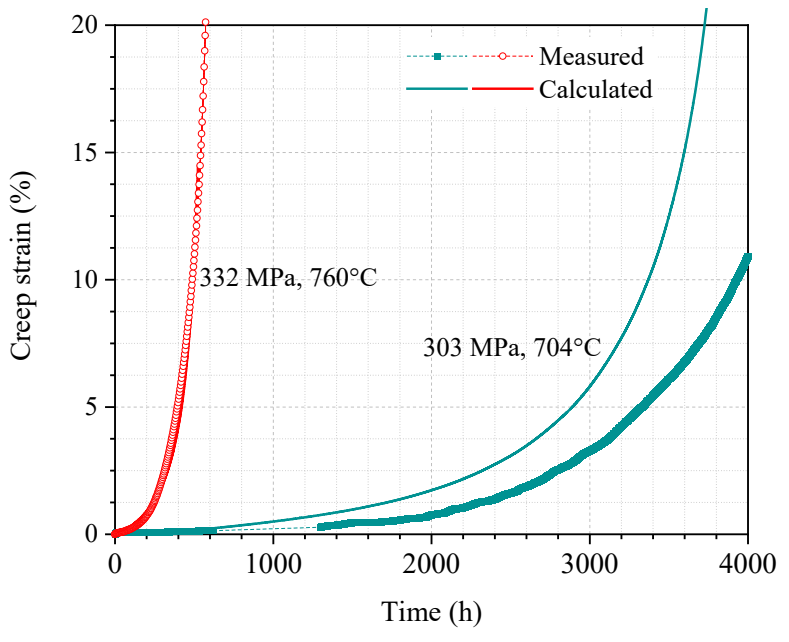

(b)

Figure 17: (a) Measured and fit creep strains (Eq. 1) for 282 and (b) comparison between measured and predicted creep strains for 282 (calculated with the parameter set estimated by fitting solution of Eq .4 to measure data shown in Figure 17a).

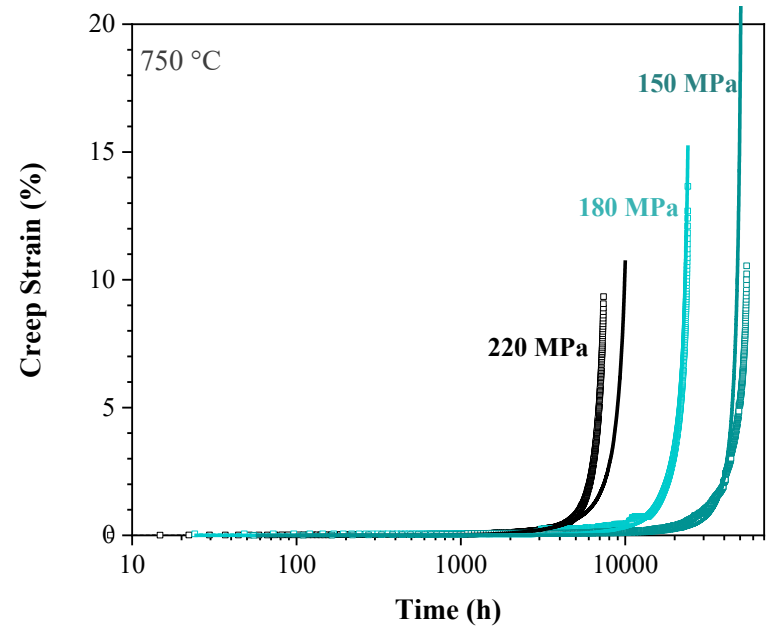

(a)

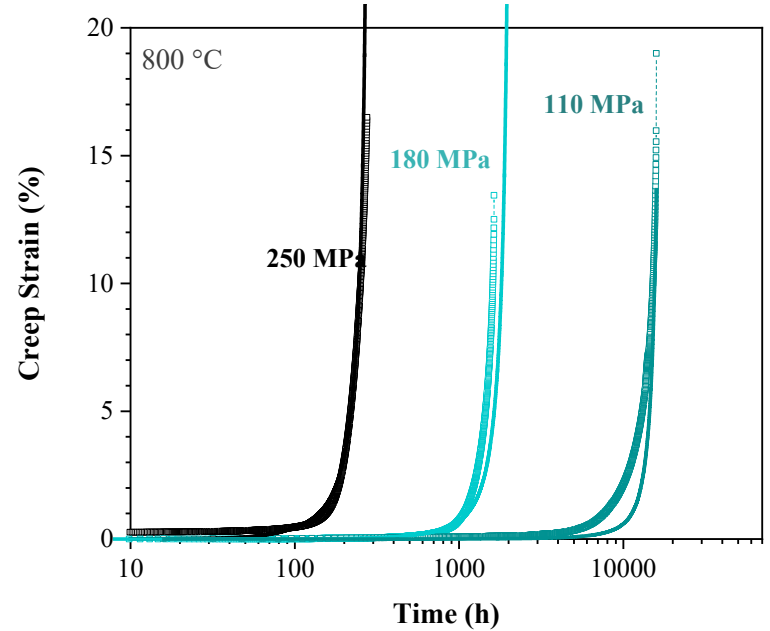

(b)

Figure 18: Comparison between measured and calculated creep strains of $740 \mathrm{H}$ at 750 and $800{ }^{\circ} \mathrm{C}$.

\section{Milestone 1.2.1 Completion of model development to perform computational validation}

Models to predict the corrosion induced degradation in $\mathrm{sCO}_{2}(\mathrm{Cr}$-depletion and metal loss) and in molten chloride salts (Cr-depletion and depth of attack) that capture the underlying physics of the degradation processes were implemented. Although the goals set in the project required a relatively simple description of the Larson-Miller parameter to predict the stress-temperature dependence of creep rupture, additional work was performed to accommodate the feedback from industry. Based on input from Brayton Energy, the time to a designated creep strain of $1-2 \%$ is a more relevant failure criteria than time to creep rupture. With this in mind, a CDM modeling approach was developed to describe the complete creep behavior of the three candidate alloys. The model integrated potential creep degradation mechanisms to enable the predictions of time 
to a designated strain (failure criteria provided by industry). Test calculations were performed with both the corrosion and creep models and the modeling results were compared with experimental data. The corrosion model was able to predict the experimental observations in terms of $\mathrm{Cr}$ depletion and depths of attack with a good accuracy $( \pm 15 \%$ of statistical variations and measurement errors). The creep model predicted the time to $2 \%$ strain with an acceptable accuracy $( \pm 500 h)$.

\section{Task 3: Computational validation}

\subsection{Subtask 3.1: Compare Calculated and Measured $\mathrm{Cr}$ Concentration at Oxide-Alloy Interface}

\subsubsection{Corrosion in $\mathrm{sCO}_{2}$}

The $\mathrm{Cr}$ concentration at the oxide/alloy interface is required to be above $10 \mathrm{wt} \%$ in $\mathrm{Ni}$ based alloys to ensure formation of a protective chromia scale [13, 45]. This is considered as corrosion induced lifetime for exposures in $\mathrm{sCO}_{2}$. Hence, it is essential to predict this value as a function of time and temperature.

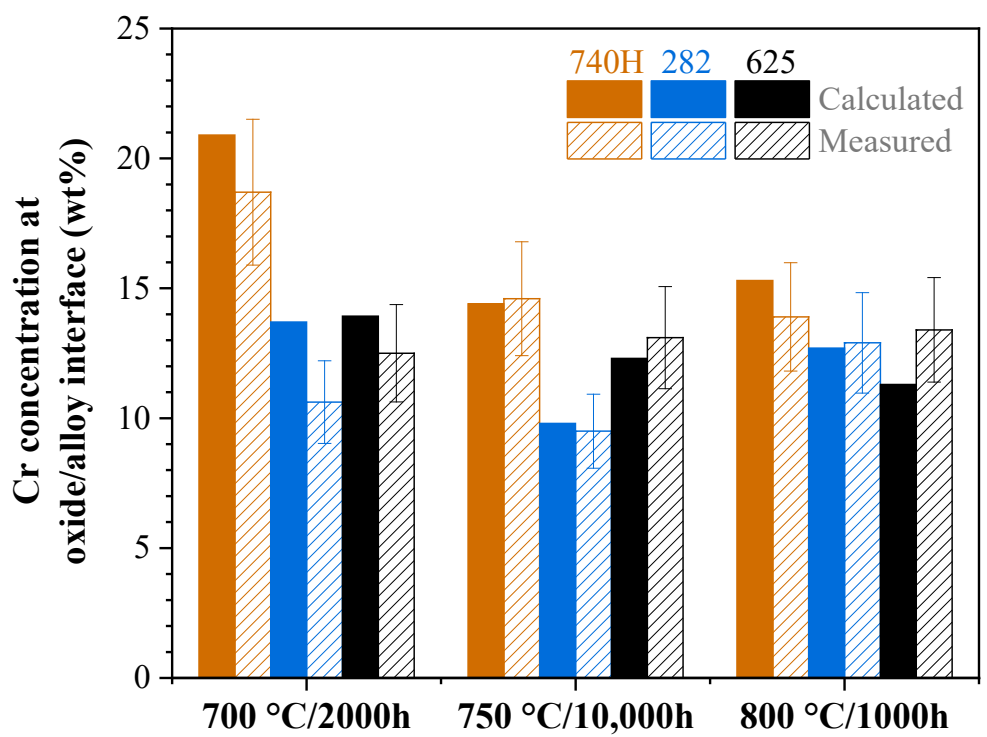

Figure 19: Comparison between measured (EDS) and calculated $\mathrm{Cr}$ concentrations at the oxide/alloy interface in $740 \mathrm{H}, 282$ and 625 after exposure in $\mathrm{sCO}_{2}$ at different times at 700,750 and $800{ }^{\circ} \mathrm{C}$.

Whiskers on measurements represent 1 standard deviation.

Figure 19 compares the measured (EDS) and calculated $\mathrm{Cr}$ concentrations at the oxide/alloy interface in $740 \mathrm{H}, 282$ and 625 after exposure in $\mathrm{sCO}_{2}$ at the maximum exposure times 700,750 and $800{ }^{\circ} \mathrm{C}$. Additional calculations are shown in Figure 20 that compare the measured (EDS) and calculated $\mathrm{Cr}$ concentrations at the oxide/alloy interface in $740 \mathrm{H}, 282$ and 625 after exposure in $\mathrm{sCO}_{2}$ at different times at 700,750 and $800^{\circ} \mathrm{C}$. All predicted values are within $\pm 10 \%$ (1 standard deviation) of typical statistical variation in corrosion tests and EDS measurement errors $( \pm 0.5 \mathrm{wt} \%)$. Since, the $10 \mathrm{wt} \% \mathrm{Cr}$ at the oxide/alloy interface might be a conservative lifetime criteria, an additional lifetime criteria of $5 \mathrm{wt} \% \mathrm{Cr}$ (based on existing experimental observations) at the $\mathrm{Cr}_{2} \mathrm{O}_{3}$ /alloy interface was implemented in the model. 


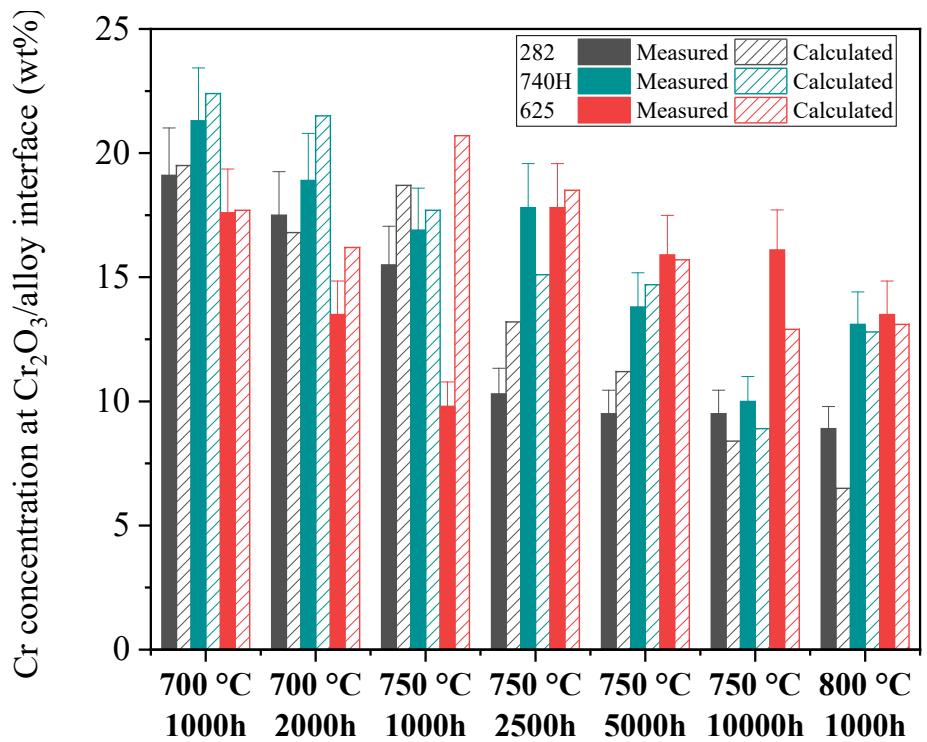

Figure 20: Comparison between measured (EDS) and calculated $\mathrm{Cr}$ concentrations at the oxide/alloy interface in $740 \mathrm{H}, 282$ and 625 after exposure in sCO2 at different times at 700,750 and $800{ }^{\circ} \mathrm{C}$. Whiskers on measurements represent 1 standard deviation.

\subsubsection{Corrosion in molten chloride salts}

For the exposures in purified molten salts, there is no protective scale formation. Material degradation is driven by selective dissolution of $\mathrm{Cr}$ from the three candidate alloys. Hence, the depth of Cr-depletion is typically equivalent to the depths of corrosion induced attack in molten chloride salts. For exposures in molten salts, this was considered as a lifetime criteria. Figure 21 shows the measured and calculated depths of attack for various Ni-based commercial alloys after exposure in purified $\mathrm{KCl}-\mathrm{MgCl}_{2}$ under different test conditions. The model predictions are within 1 standard deviation (whiskers) of the measurements in most cases.

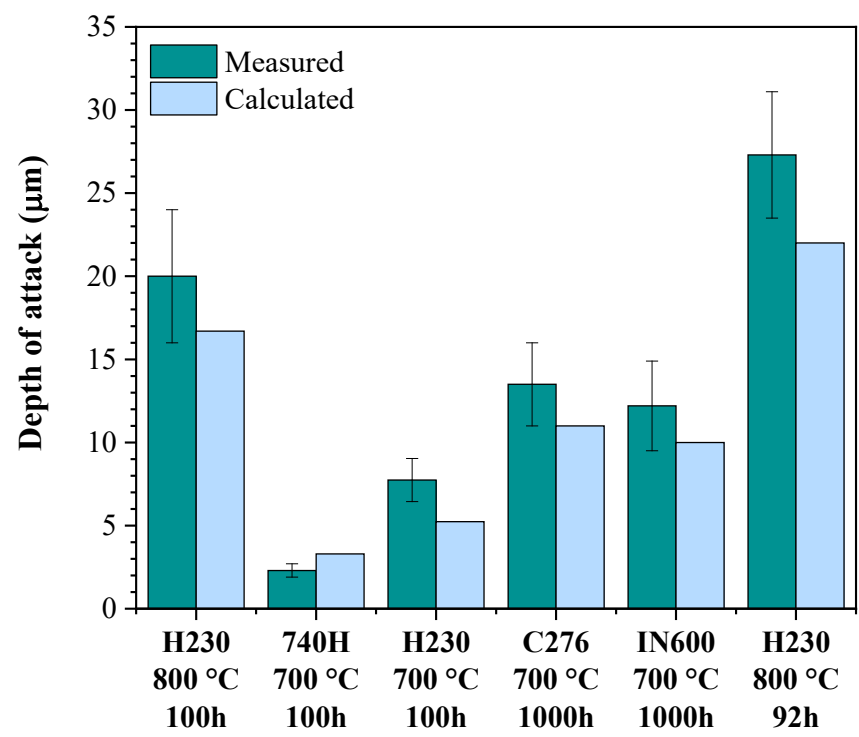

Figure 21: Comparison between measured (EDS + image analyses) and calculated depths of attack in various $\mathrm{Ni}$-based commercial alloys after exposure in purified $\mathrm{KCl}-\mathrm{MgCl}_{2}$. 
To elucidate the correlation between LMP and the phase fractions of the strengthening phases, the temperature dependence of the phase stabilities in $740 \mathrm{H}$ and 282 was calculated with the thermodynamic database TCNI8 [53]. The calculated phase fraction of $\gamma^{\prime}, \boldsymbol{\varphi}_{\gamma^{\prime}}$ in $740 \mathrm{H}$ and 282 are plotted vs the respective LMP in Figure 22a and b respectively.

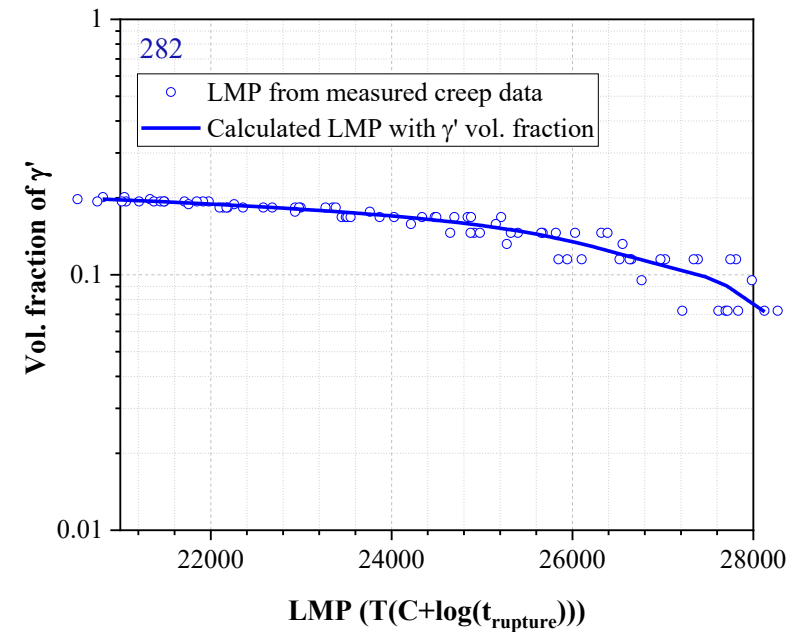

(a)

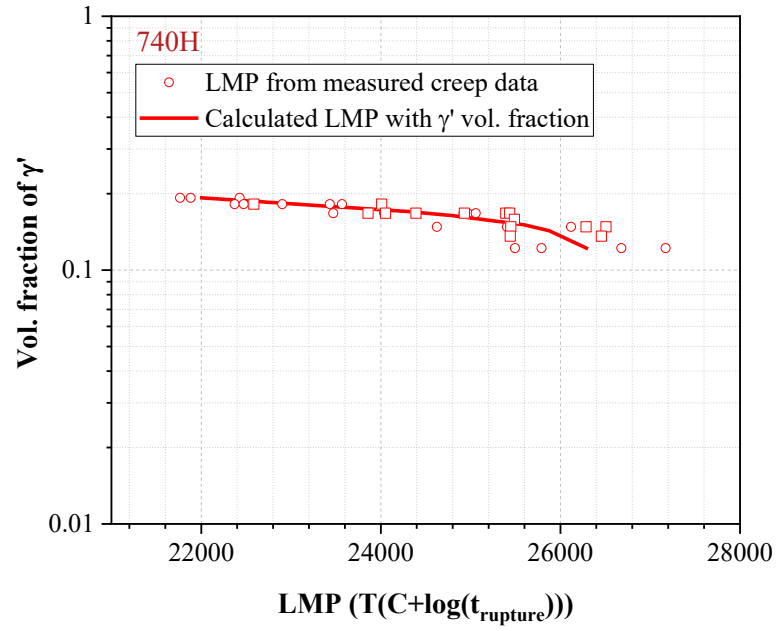

(b)

Figure 22: Correlation between LMP and vol. fraction of $\gamma^{\prime}$ in (a) 282 and (b) $740 \mathrm{H}$. Symbols correspond to measured data for LMP and solid line represents calculated LMP with the vol. fraction of $\gamma^{\prime}$.

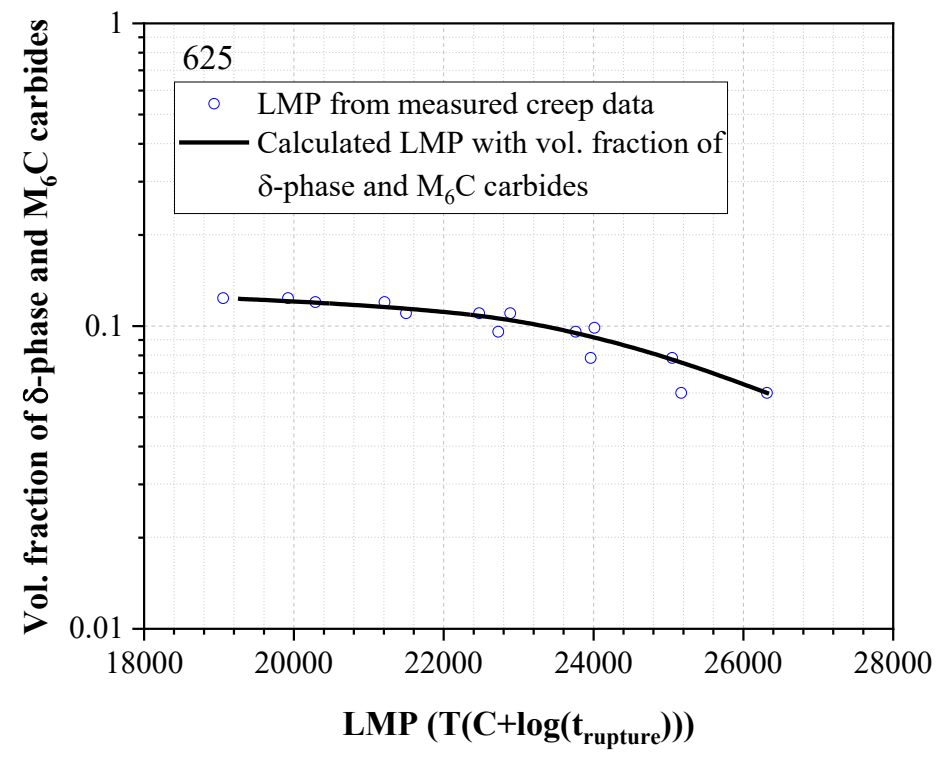

Figure 23: Correlation between LMP and vol. fraction of $\delta$-phase and $\mathrm{M}_{6} \mathrm{C}$ carbides in 625 . Symbols correspond to measured data for LMP and solid line represents calculated LMP with the vol. fraction of $\delta$-phase and $\mathrm{M}_{6} \mathrm{C}$ carbides.

The influence of stress on the dissolution of $\gamma^{\prime}$ is not known (and cannot be derived from existing data since this would require extensive microstructural characterization) but there is a clear correlation between $\varphi_{\gamma^{\prime}}$ and LMP. The solid lines in Figure 22a and b represent the LMP values calculated with $\varphi_{\gamma^{\prime}}$ with a function of the form $a+b \cdot \log \left(\varphi_{\gamma^{\prime}}\right)+c \cdot \log \left(\varphi_{\gamma^{\prime}}\right)^{2}+d \cdot \log \left(\varphi_{\gamma^{\prime}}\right)^{3+}$ $e \cdot \log \left(\varphi_{\gamma^{\prime}}\right)^{4}$, whereby the parameters a-e were estimated by fitting to measured data. Although 
additional mechanisms (discussed under Creep modeling) most likely contribute to the overall creep behavior, the demonstrated relation between $\varphi_{\gamma^{\prime}}$ and LMP suggests that the depletion of $\boldsymbol{\varphi}_{\gamma^{\prime}}$ is a key degradation creep mechanism in $740 \mathrm{H}$ and 282.

In the case of the solid solution strengthened alloy 625 , the creep degradation mechanisms have not been unambiguously identified in the literature. The presence of additional intermetallic precipitates such as $\delta-\mathrm{Ni}_{3} \mathrm{Nb}$ phase and (Mo,Si)-rich $\mathrm{M}_{6} \mathrm{C}$-type carbides might additionally be contributing to the creep strength of 625 [64]. The temperature dependence of sum of phase fractions of $\delta-\mathrm{Ni}_{3} \mathrm{Nb}$ phase and $\mathrm{M}_{6} \mathrm{C}$ carbides $\boldsymbol{\varphi}_{\delta+\mathrm{M}_{6} \mathrm{C}}$ was calculated with the thermodynamic database TCNI8 [53]. As in the case of $740 \mathrm{H}$ and 282, a correlation between $\boldsymbol{\varphi}_{\delta+\mathrm{M}_{6} \mathrm{C}}$ and LMP is evident in Figure 23.

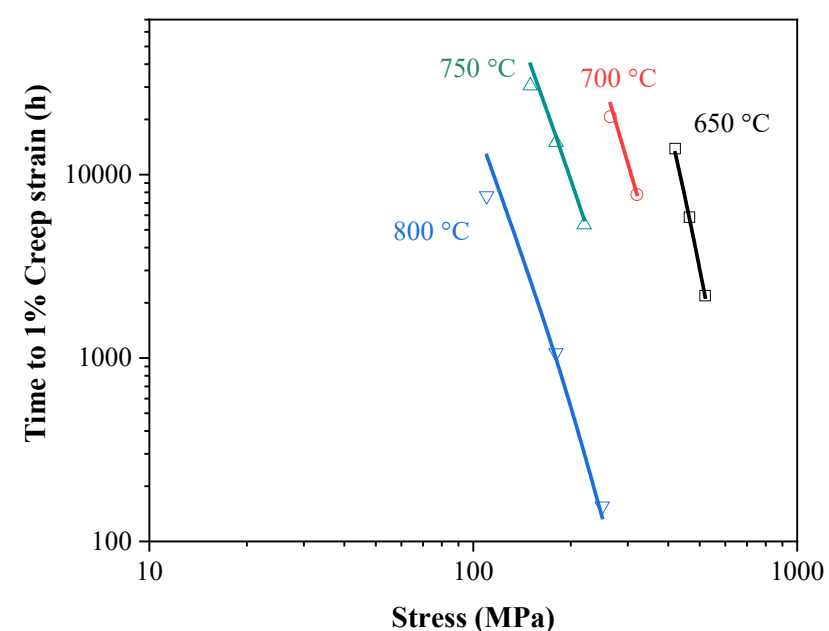

(a)

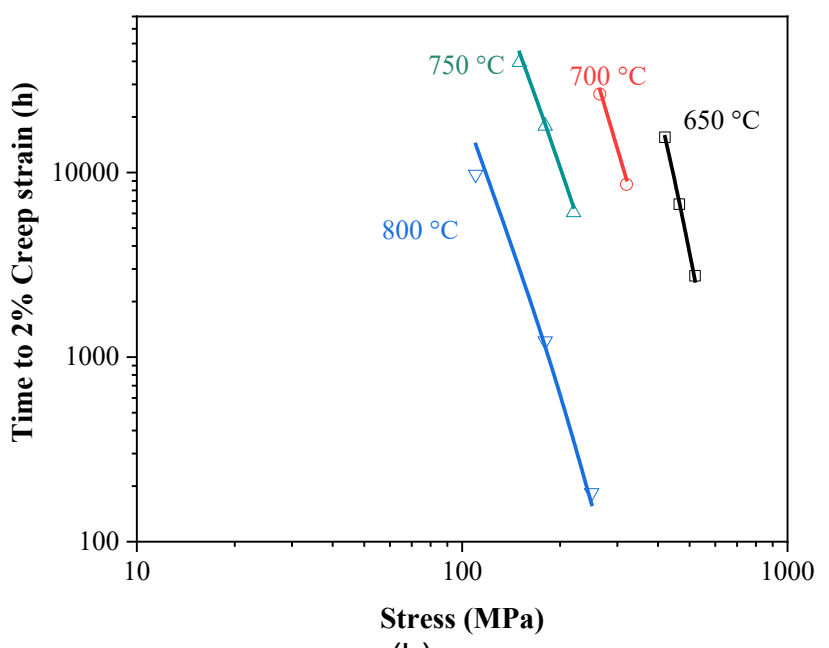

(b)

Figure 24: Comparison between measured (symbols) and calculated (lines) times to (a) $1 \%$ and (b) $2 \%$ creep strains for $740 \mathrm{H}$.

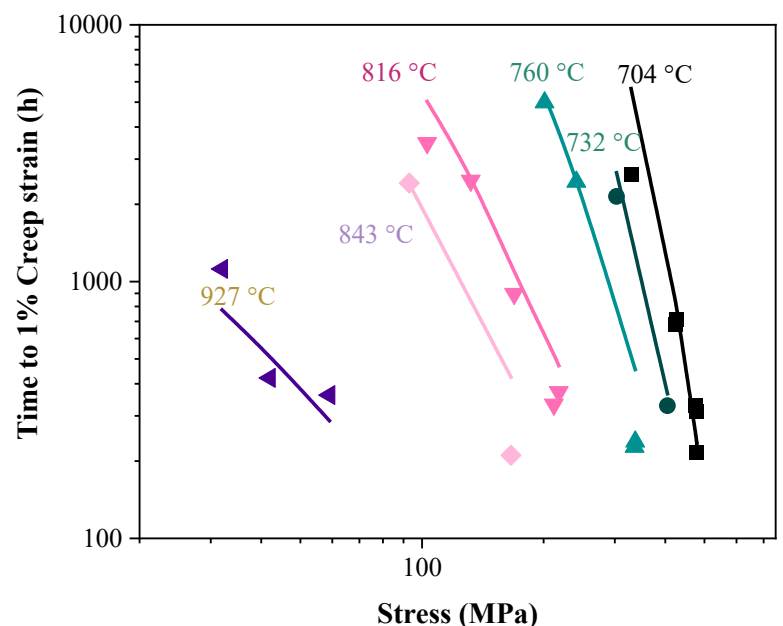

(a)

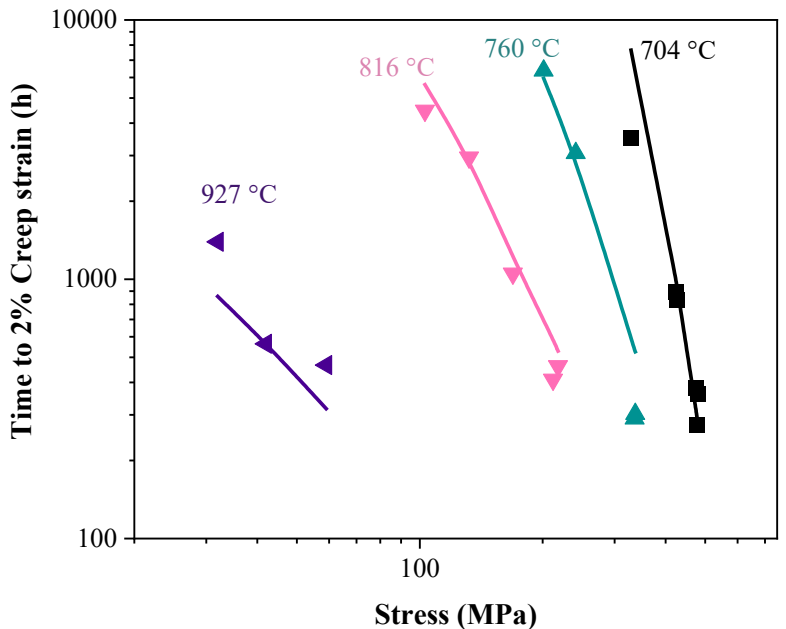

(b)

Figure 25: Comparison between measured (symbols) and calculated (lines) times to (a) $1 \%$ and (b) $2 \%$ creep strains for 282.

In addition to identifying the correlation between LMP and the fraction of strengthening precipitate phases, the times to 1 and $2 \%$ creep strains $t_{2 \%}$ (failure criteria) were also calculated with the CDM model described earlier. Figure $24 a$ and b compare the measured and calculated 
times to 1 and $2 \%$ creep strain for $740 \mathrm{H}$ respectively. A similar comparison is given for 282 in Figure 25. Given the limited data and microstructural information, the CDM model was able to predict $t_{2 \%}$ with an accuracy of $\pm 500 \mathrm{~h}$.

\section{Task 4: Generate screening protocol for currently immature materials}

The goal of this task was to review existing literature data for $\mathrm{SiC}$ to identify differences in failure mechanisms between $\mathrm{SiC}$ and FelNi-base alloys. The quality and variability of the existing data were evaluated. The gaps in experimental data were identified and other considerations will be evaluated for integration into the model. Recommendations were made for future development of the tool to include SiC.

\subsubsection{Subtask 4.1: Review existing literature data for one chosen sample material}

\subsubsection{Corrosion in $\mathrm{sCO}_{2}$}

There is limited data on corrosion behavior of $\mathrm{SiC}$ in $\mathrm{sCO}_{2}$ [65-70]. Sridharan et al. [68] reported minimal mass gain after an initial mass loss for SiC after exposure for 1000h in research grade $\mathrm{sCO}_{2}$ at $750^{\circ} \mathrm{C}$. The authors attributed the initial mass loss to the removal of $\mathrm{C}$ from $\mathrm{SiC}$ in the early stages of exposure which was partially supported by the observation of pits within the $\mathrm{SiC}$ matrix. After the initial mass loss, formation of silicon oxide was observed on the surface and within SiC. Walker et al. [69] exposed Hexoloy $\mathrm{SiC}$ in $\mathrm{CO}_{2}$ at $900{ }^{\circ} \mathrm{C}$ for up to $2000 \mathrm{~h}$ and reported similar results as Sridharan et al. [68] with initial mass loss up to $1000 \mathrm{~h}$ and then slight mass gains thereafter. The measured mass changes for $\mathrm{SiC}$ exposed at $900{ }^{\circ} \mathrm{C}$ were minimal compared to the Ni-base alloys 230, 282 and $740 \mathrm{H}$ exposed at $900{ }^{\circ} \mathrm{C}$ in $\mathrm{CO}_{2}$ suggesting good compatibility of $\mathrm{SiC}$ in high temperature $\mathrm{CO}_{2}$ environments. Pint et al. [71] tested CVD $\mathrm{SiC}$ in 200 bar $\mathrm{CO}_{2}$ at 650,700 and $750^{\circ} \mathrm{C}$ for $500 \mathrm{~h}$. Negligible mass change was observed at all three temperatures compared to $\mathrm{Fe}$ - and Ni-based alloys. Bremen et al. [72] investigated the corrosion behavior of pyrolytically deposited $\beta-\mathrm{SiC}$ in various $\mathrm{CO}-\mathrm{CO}_{2}$ mixtures $\left(5-70\right.$ vol\% $\left.\mathrm{CO}_{2}\right)$ between 1000 and $1400{ }^{\circ} \mathrm{C}$. An external $\mathrm{SiO}_{2}$ layer was observed in all cases. The oxidation rate was governed by the inward transport of $\mathrm{O}$ in $\mathrm{SiC}$. Existing results in the literature suggest good compatibility of $\mathrm{SiC}$ in $\mathrm{CO}_{2}$.

\subsubsection{Corrosion in molten halide salts}

There is even less data for corrosion behavior of $\mathrm{SiC}$ in molten chloride salts. All available data in molten chloride and fluoride salts have been summarized here. Nagaoka et al. [73] studied the corrosion of $\mathrm{SiC}$ in molten $\mathrm{KCl}-\mathrm{NaCl} \mathrm{SiC}$ sintered with $\mathrm{B}$ and $\mathrm{C}$ were immersed in as-received $\mathrm{KCl}-\mathrm{NaCl}$ in lidded alumina crucibles at 700 and $800{ }^{\circ} \mathrm{C}$ up to $400 \mathrm{~h}$. The authors did not report mass change data or perform cross-section analyses to report depths of attack. $\mathrm{SiO}_{2}$ was observed as a corrosion product on the specimen surfaces and was accompanied by significant roughening of the surface.

Espinoza-Perez et al. [74] evaluated the influence of microstructure of monolithic SiC on corrosion rates in molten FLiNaK salt at $900{ }^{\circ} \mathrm{C}$ for $250 \mathrm{~h}$. Assuming linear corrosion rates, the authors concluded that the corrosion rate increased with the density of high angle grain boundaries (HAGB) suggesting that these boundaries were most susceptible to corrosion. In contrast, the corrosion rates decreased with increasing density of low angle grain boundaries (LAGB). However, it must be mentioned that the FLiNaK salt wasn't purified and the influence of impurities on the corrosion rates was not examined. 
Walker et al. [67] exposed $\mathrm{SiC}$ in a binary $\mathrm{KCl}-\mathrm{MgCl}_{2}(62.5-37.5 \mathrm{wt} . \%)$ mixture for up to $503 \mathrm{~h}$ at $750^{\circ} \mathrm{C}$. A large variability in mass change data was observed for the SiC specimens and no particular reason was provided by the authors. A comparison with $\mathrm{H} 230$ was provided for a single specimen after exposure for $108 \mathrm{~h}$ and a significantly lower mass loss was observed for $\mathrm{SiC}$ $\left(\sim 0.03 \mathrm{mg} \mathrm{cm}^{-2}\right)$ than for $\mathrm{H} 230\left(\sim 2.75 \mathrm{mg} \mathrm{cm}^{-2}\right)$.

Gu et al. [75] reported that $\mathrm{SiC}$ corroded in molten FLiNaK salt at $800^{\circ} \mathrm{C}$ via the dissolution of $\mathrm{Si}$ in the salt resulting in the formation of a carbon-rich surface. The decreasing corrosion rate of $\mathrm{SiC}$ with increasing exposure time was attributed to the potentially protective $\mathrm{C}$-rich layer on the surface.

\subsubsection{Subtask 4.2: Identify key degradation mechanisms of chosen material}

With the limited data on the corrosion behavior of $\mathrm{SiC}$ in $\mathrm{sCO}_{2}$ and molten halide salts, it is difficult to conclusively identify corrosion induced degradation of $\mathrm{SiC}$ in these media at temperatures of interest $\left(600-800{ }^{\circ} \mathrm{C}\right.$ ). With the data available for corrosion of $\mathrm{SiC}$ in $\mathrm{CO}_{2}$ (mainly at temperatures $>1200^{\circ} \mathrm{C}$ ) [76], $\mathrm{SiC}$ is expected to oxidize according to the following reaction in $\mathrm{CO}_{2}$ :

$$
\mathrm{SiC}+3 \mathrm{CO}_{2}(\mathrm{~g})=\mathrm{SiO}_{2}+4 \mathrm{CO}(\mathrm{g})
$$

Opila and Nguyen [76] have shown that the oxidation rates of $\mathrm{SiC}$ in high purity $\mathrm{CO}_{2}$ are about 2-3 orders of magnitude lower than in $\mathrm{O}_{2}$ or $\mathrm{H}_{2} \mathrm{O}$ at $1200{ }^{\circ} \mathrm{C}$. The results reported by Sridharan et al. [68], Walker et al. [69] and Pint et al. [71] provide additional evidence that SiC shows very low mass changes during exposures in $\mathrm{sCO}_{2}$ and $\mathrm{CO}_{2}$ respectively. It must be however mentioned that except for the results presented by Sridharan et al. [68] and Pint et al. [71], none of the testing performed by other authors was under high pressure. There is a significant lack of data in this regard.

Although existing literature suggests the better corrosion resistance of $\mathrm{SiC}$ in molten halide salts, there are other factors to consider. Studies have shown that intentionally added impurities (such as $\mathrm{Ni}, \mathrm{Cr}$ ions, etc.) to molten FLiNaK salt, led to a higher mass loss and increased corrosion for SiC $[77,78]$. Yang et al. reported the possibility of Ni reacting with $\mathrm{Si}$ in the salt to form NiSi and $\mathrm{Ni}_{31} \mathrm{Si}_{12}$ on the surface of Hastelloy $\mathrm{N}$ alloy that can drive the dissolution of $\mathrm{Si}$ from $\mathrm{SiC}$ into salt and alloy. Corrosion products such as $\mathrm{CrF}_{2}$ can further drive corrosion by forming Cr-rich carbides on the $\mathrm{SiC}$ surface and consequently dissolving $\mathrm{Si}$ from $\mathrm{SiC}$ into the salt. The effect of $\mathrm{SiC}$ microstructure on the corrosion and susceptibility to form localized attack are additional factors to consider [79].

No definite conclusions can be drawn on the long-term impact of selective dissolution of $\mathrm{Si}$ in the molten halide salts, as reported by Gu et al. [75]. Currently there are no long-term data available in the literature. However, it can be hypothesized that the protective nature of the C-rich layer [75] is compromised by the presence of metallic elements with high affinity for $\mathrm{C}$ (e.g., $\mathrm{Cr}$, $\mathrm{Ti}, \mathrm{Nb}, \mathrm{W}$ ). This might dissolve the $\mathrm{C}$-rich layer by forming carbides and reinitiate the dissolution of Si.

\subsubsection{Degradation of mechanical properties}

Monolithic SiC typically shows brittle fracture and hence SiC/SiC composites are considered better candidates as structural materials for high temperature applications [70]. In the view of fracture mechanics of composites, the fiber-matrix interface is the most important factor that determines the structural stability of a composite. Intensive efforts have been devoted to study the influences of interface on mechanical properties of $\mathrm{SiC}-\mathrm{SiC}$ composites [80-83]. A monolithic ceramic test specimen under a tensile load will fail when a sufficiently large flaw initiates a crack that unstably propagates through the gage section. Unlike monolithic ceramics, 
however, damage and failure in continuous fiber CMCs do not result from the propagation of a single crack. Instead, small cracks initiate throughout the matrix because shear at the fiber-matrix interface facilitates the transmission of applied load to the fibers. In the vicinity of a matrix crack, load is shed from the matrix to the fiber. Each crack grows until the matrix is saturated and no more matrix cracks develop. Because cracks flood the matrix prior to failure, a CMC can exhibit nominal strains that are orders of magnitude larger than its monolithic counterpart and, like a metal, it can exhibit non-linear deformation and non-catastrophic failure [84].

The high toughness and non-linear deformation of continuous fiber CMCs result from a weakly bonded fiber-matrix interface. The fiber coating in the $\mathrm{SiC} / \mathrm{SiC}$ system has a lower elastic modulus and lower tensile strength than both the fiber and matrix. This produces a weak fibermatrix interface that encourages cracks to propagate around fibers rather than through them, a phenomenon called crack deflection. Crack deflection allows fibers in the wake of a crack to remain intact and exert fiber bridging tractions that prevent the crack from growing unstably. Above and below the fracture plane, portions of the fiber-matrix interface debond; the length over which the interface debonds is known as the debond length. As a fiber is stretched, portions along the debond length slide against the matrix, dissipating energy as a result of frictional shear.

Thus far two mechanisms of crack resistance have been discussed - crack deflection and frictional sliding. The toughness of CMCs is attributed to these mechanisms, as well as widespread cracking. This is best illustrated using the example of a unidirectional laminate loaded in tension with fibers oriented in the loading direction. As load is applied, the composite first deforms elastically, with deformation becoming non-linear at or after matrix crack initiation. Periodically spaced matrix cracks accumulate throughout the gage length of the laminate (generally oriented normal to the loading direction) up to a point of saturation, which is characterized by uniformly spaced matrix cracks. Fibers eventually fracture with continued loading; the distance between the fiber fracture location and the nearest crack plane is called the pullout length. Frictional sliding along this length dissipates energy, allowing for failure. Based on the literature findings, it is clear that the structural damage mechanisms in $\mathrm{SiC}$ and $\mathrm{SiC} / \mathrm{SiC}$ composites are not completely similar to Ni-based alloys and the key differences are:

1. Brittle failure

2. Dependence of failure on the fiber-matrix interface

3. Lack of strengthening phases in SiC

However, creep in $\mathrm{SiC}$ and $\mathrm{SiC} / \mathrm{SiC}$ composites is governed by dislocation climb [85] and can be described accordingly if relevant experimental creep data is available.

\subsubsection{Subtask 4.3: Obtain information on required model parameters to describe degradation and predict lifetime of chosen material}

\subsubsection{Oxidation lifetime in $\mathrm{sCO}_{2}$}

The oxidation of $\mathrm{SiC}$ can be modeled based on prior works [86-88]. The simultaneous oxidation of $\mathrm{Si}$ and evaporation of $\mathrm{SiO}$ (at low partial pressures of oxygen) and $\mathrm{SiO} 2$ (at high temperatures) need to be considered to accurately model the oxidation induced degradation of $\mathrm{SiC}$. The influence of moisture on the enhanced oxygen permeation and on the reactive evaporation of scale in the form of $\mathrm{Si}(\mathrm{OH})_{4}$ can be ignored for exposures in $\mathrm{sCO}_{2}$. In flowing gases, the effect of external fluid velocity on the inward gas-phase diffusion between the ambient and scale surface of $\mathrm{O}_{2}$ and outward diffusion of $\mathrm{SiO}_{2}$, and $\mathrm{SiO}$ can be included [89] but these rates are significantly low at temperatures of interest $\left(700-800{ }^{\circ} \mathrm{C}\right)$. Gas-phase multicomponent diffusivities within the boundary layer can be modeled based on work by Parthasarathy et al. [90]. 
The following steps are adopted to model the oxidation of $\mathrm{SiC}$ and the consequent recession of the $\mathrm{SiC}$ surface. A description of all the variables and their sources are provided in Table 7.The partial pressure of oxygen at the gas/scale interface is calculated as,

$$
P_{\boldsymbol{O}_{2}}^{\boldsymbol{s}}=\frac{\boldsymbol{D}_{\mathrm{O}_{2}} \boldsymbol{l}_{\mathrm{scale}} \boldsymbol{P}_{\mathrm{O}_{2}}^{\mathrm{a}}}{\boldsymbol{D}_{\mathrm{O}_{2}} \boldsymbol{l}_{\text {scale }}+\boldsymbol{\delta}_{\mathrm{bl}} \boldsymbol{R T} \boldsymbol{\alpha}_{02 \text {-scale }}}
$$

The boundary layer thickness is $\delta_{\mathrm{bl}}$ calculated as,

$$
\delta_{\mathrm{bl}}=1.5\left(\frac{\eta}{\rho D_{\mathrm{O}_{2}}}\right)^{-1 / 3}\left(\frac{\rho V L}{\eta}\right)^{-1 / 2}
$$

Oxidation-induced recession of the SiC surface is then calculated as

$$
\frac{\mathrm{d} X_{S i C}}{\mathrm{~d} t}=\frac{2}{3} \mathrm{~V}_{\mathrm{SiC}} \alpha_{\text {O2-scale }} \frac{P_{O_{2}}^{S}}{l_{\text {scale }}}
$$

\subsubsection{Corrosion lifetime in molten chloride salts}

As discussed under Subtask 4.1, the lack of data for corrosion of $\mathrm{SiC}$ in molten chloride salts is a barrier in development of mechanistic models to describe the underlying corrosion processes. A detailed understanding of the interaction between $\mathrm{SiC}$ and molten chloride salts is essential to be able to quantify the extent of corrosion induced degradation. Furthermore, the composite nature of the material suggests a much stronger dependence of corrosion behavior on the microstructure, as shown by Espinoza-Perez et al. [67]. Typically, analogous to the dissolution of $\mathrm{Cr}$ from Ni-based alloys, the dissolution of $\mathrm{Si}$ in the molten halide salts will most likely govern the corrosion kinetics.

Table 7: Description of the parameters for the model to predict oxidation lifetime of SiC

\begin{tabular}{|c|c|c|c|}
\hline Symbol & Units & Description & Sources \\
\hline$P_{\mathrm{O}_{2}}^{s}$ & $\mathrm{~Pa}$ & partial pressure of oxygen at the gas/scale interface & Calculated \\
\hline$P_{\mathrm{O}_{2}}^{\mathrm{a}}$ & $\mathrm{Pa}$ & Ambient partial pressure of oxygen & Input \\
\hline$D_{\mathrm{O}_{2}}$ & $\mathrm{~m}^{2} / \mathrm{s}$ & Diffusivity of O in the boundary layer & {$[91]$} \\
\hline$l_{\text {scale }}$ & $\mathrm{m}$ & Thickness of oxide scale & Calculated \\
\hline$\delta_{\mathrm{bl}}$ & $\mathrm{m}$ & Thickness of boundary layer & Calculated \\
\hline$\alpha_{02-\mathrm{scale}}$ & $\begin{array}{c}\mathrm{mol} / \mathrm{m}- \\
\mathrm{s}-\mathrm{Pa}\end{array}$ & Permeability coefficient of O in SiO 2 & {$[91]$} \\
\hline$\eta$ & $\mathrm{Pa}-\mathrm{s}$ & viscosity of O in boundary layer & {$[92]$} \\
\hline$\rho$ & $\mathrm{kg} / \mathrm{m}^{3}$ & Density of O in the boundary layer & {$[92]$} \\
\hline$V$ & $\mathrm{~m} / \mathrm{s}$ & Velocity of ambient fluid & Input \\
\hline $\mathrm{L}$ & $\mathrm{m}$ & Length of component & Input \\
\hline $\mathrm{V}_{\mathrm{SiC}}$ & $\mathrm{m}^{3} / \mathrm{mol}$ & Molar volume of SiC & Input \\
\hline
\end{tabular}

This can result in compositional changes near the alloy surface, e.g., enrichment of $C$ on the alloy surface, as demonstrated by Gu et al. [75] for SiC exposures in molten FLiNaK at 800 ${ }^{\circ} \mathrm{C}$. Under these conditions, the corrosion rate will be governed by the transport of $\mathrm{Si}$ in $\mathrm{SiC}$. If the diffusion coefficients of $\mathrm{Si}$ and $\mathrm{C}$ in $\mathrm{SiC}$ are available at the temperatures of interest, the depletion of $\mathrm{Si}$ and depth of attack can be calculated, as has been implemented in the tool developed here to simulate the depletion of $\mathrm{Cr}$ from alloys $740 \mathrm{H}, 282$ and 625 during high temperature exposures 
in molten $\mathrm{KCl}-\mathrm{MgCl}_{2}$. It must be mentioned here that the data for diffusion coefficients in the literature demonstrate considerable scatter mainly due to the strong structure dependence of transport mechanisms [93]. With reliable experimental data for $\mathrm{SiC}$ in the desired halide salt, the diffusion coefficients can be estimated by fitting to the measured compositional profiles for Si and C. Corrosion lifetimes can be predicted with this information and solution of diffusion equations.

\subsubsection{Mechanical lifetime}

Various modeling approaches have been proposed in the literature to predict the time dependent structural failure of $\mathrm{SiC} / \mathrm{SiC}$ composites [94, 95]. One of these methods presented by Lara-Curzio [96] relevant for the operating temperatures of interest here will be elucidated. Creep can be ignored since $\mathrm{SiC}$ is expected to behave elastically at temperatures between $700-800{ }^{\circ} \mathrm{C}$ [85]. The model also accounts for the oxidation induced degradation of the fibers in the composite material. A description of all the variables and their sources are provided in Table 8.

Table 8: Description of the parameters for the model to predict mechanical lifetime of SiC

\begin{tabular}{|c|c|c|c|}
\hline Symbol & Units & Description & Source \\
\hline$\Phi$ & - & Failure probability of the composite material & Calculated \\
\hline$l$ & $\mathrm{~m}$ & Fiber gauge length & Input \\
\hline$l_{0}$ & $\mathrm{~m}$ & Reference gauge length & Material property \\
\hline$N_{0}$ & - & Total number of fibers & Input \\
\hline$N$ & $\mathrm{~m}$ & Number of undamaged fibers & Calculated \\
\hline$E$ & $\mathrm{GPa}$ & Elastic modulus of the fiber & Material property \\
\hline$K_{\mathrm{IC}}$ & $\mathrm{MPa} \mathrm{m} \mathrm{m}^{0.5}$ & Fracture toughness & Material property \\
\hline$Y$ & - & Geometric parameter & Material property \\
\hline $\mathrm{L}$ & $\mathrm{m}$ & Length of component & Input \\
\hline $\mathrm{V}_{\mathrm{SiC}}$ & $\mathrm{m}^{3} / \mathrm{mol}$ & Molar volume of SiC & Input \\
\hline
\end{tabular}

The model assumes that the structural property of a single fiber can be employed to describe the mechanical behavior (e.g., same linear stress-train behavior) of the composite material. It is further assumed that the strength controlling flaws are restricted to the surface of the fibers. Typically, the strength of the fibers is described by a two-parameter Weibull distribution and the probability of failure of the fibers $\Phi$ when subjected to a uniform tensile stress $\sigma$, is given by

$$
\Phi=1-\exp \left(-\frac{l}{l_{0}}\left(\frac{\sigma}{\sigma_{0}}\right)^{m}\right)=1-\frac{N}{N_{0}}
$$

The stress $\sigma$ on each fiber under an applied load $F$ is then given by,

$$
\sigma=\frac{F}{A N}
$$

while the strain on each fiber $\varepsilon$ can be calculated as,

$$
\varepsilon=\frac{\sigma}{E}
$$

The time dependent fiber characteristics strength beyond the time when the thickness of the oxide layer growing on the fiber $X_{\mathrm{SiO} 2}$ (estimated from the oxidation model described above) is greater than a critical flaw size can then be calculated as,

$$
\sigma_{0}(t)=\frac{K_{\mathrm{IC}}}{Y \sqrt{X_{\mathrm{SiO2}}}}
$$


The stress-strain response of the material can be calculated with Eqs. 14 and 15 and the failure with Eq. 16. The time dependent failure probability for the composite material can be calculated by combining Eqs. 13 and 16 .

\section{M (T-1.4.1) Document reporting the pathway for screening future materials}

The pathway for screening $\mathrm{SiC}$ has been reported under Task 4 and serves as the requested document.

\section{Task 5: Provide accessibility to modeling tool Subtask 5.1 Provide developed tool for testing to user (ORNL)}

The corrosion and creep models were implemented in a Python environment that will allow an open-source access to the scientific community and industry. As described earlier, the tool provides the corrosion and creep lifetime of the chosen materials with the user providing input conditions of stress, temperature, corrosive media (sCO2 or purified molten chloride salts). To test the functionality of the tool, it was provided to a domain expert at ORNL. Feedback was provided with respect to bugs, ease of operation and additional improvements.

\subsubsection{Subtask 5.2 Upload developed tool to GITHUB}

The developed tool was uploaded to a public GitHub platform with all source files and required input data.

\section{M (T-1.5.1) Providing access to the alloy selection and evaluation tool}

The lifetime assessment tool has been uploaded to GITHUB and access to the tool can be obtained by sending an email to pillairr@ornl.gov.

Impact ${ }^{1}$ :

The modeling tool being developed in the current project will provide thermal industry experts with a design tool for material selection and assessment. An ongoing project with Brayton Energy with the goal of characterizing and modeling elevated temperature creep and fatigue behavior for thin sheet and foil forms of $\gamma^{\prime}$ alloys $740 \mathrm{H}$ and HR282 in wrought and as-processed for folded and brazed constructions will significantly benefit from the modeling approach incorporating all relevant physics in the being developed in the current project. The tool is currently being tested for this purpose by Brayton Energy.

\section{Path forward}

The modeling tool developed in the current project provides lifetime assessment of three candidate materials $740 \mathrm{H}, 282$ and 625 for $\mathrm{sCO}_{2} /$ molten salts heat exchanger. The tool incorporated physics-based models that accounted for all relevant underlying degradation mechanisms (creep and corrosion). Reduced-order models enabled rapid simulations of realistic component lifetimes $(\sim 100 \mathrm{kh})$ while still retaining the fidelity of the complex models.

The tool lays the foundation for continuous development to include additional candidate materials, account for additional damage mechanisms (e.g., fatigue) and further applications of

\footnotetext{
${ }^{1}$ See EERE 355 FARC Section IA5
} 
interest. The methodology adopted in the current project has demonstrated the type of experimental data and approach essential to continue the development. However, the limited resources bound to a 1-year project did not allow a thorough examination of the degradation processes to verify the model assumptions. Furthermore, any gaps in experimental data could not be complemented with additional experiments. The literature review for SiC clearly identified the gaps in experimental data for currently immature materials and the challenges in developing design protocols to enable application of these materials for CSP systems.

The procedure demonstrated in the current project can be employed to develop a universal design framework for CSP systems that will include a wider range of applications (and operating conditions) and corresponding materials. The framework will allow integration of various modeling procedures to address all relevant degradation mechanisms based on the chosen application. However, this must be supported by a supporting task for experimental evaluation and microstructural characterization to complement existing data and provide validation for model predictions. This effort can involve multiple national labs and synergistically combine the available expertise across various domains. An example for such a project can be the integration of the computational approach developed at ANL to design solar receivers and the tool developed in the current project. An extended effort (3 years) with involvement of industry partners can be extremely valuable in establishing the universal design framework.

\section{Acknowledgments}

This material is based upon work supported by the U.S. Department of Energy's Solar Energy Technology Office under the FY21 Lab Call (award number 37370) to Oak Ridge National Laboratory. Microstructural characterization was performed by T. M. Lowe, and V. Cox. Relevant experimental data for model development was provided by X. Chen, P. Tortorelli and B.A. Pint. Extremely valuable industry input was provided by Brayton Energy, LLC and Echogen. Code development was assisted by M. Romedenne and S.K. Lee at ORNL. 


\section{References}

1. Barua, B., M.C. Messner, and M.D. McMurtrey, Comparison and Assessment of the Creepfatigue and Ratcheting Design Methods for a Reference Gen3 Molten Salt Concentrated Solar Power Receiver, Proceedings of the Asme Pressure Vessels and Piping Conference, 2019, Vol 3, 2019.

2. Wright, I., S. Kung, and J. Shingledecker, Predicting the Oxidation/Corrosion Performance of Structural Alloys in Supercritical $C O<s u b>2</ s u b>$. 2017, ; Electric Power Research Inst. (EPRI), Charlotte, NC (United States). p. Medium: ED; Size: 206 p.

3. Pint, B.A. and R.R. Pillai, Lifetime Model Development for Supercritical CO2 CSP Systems. 2019, ; Oak Ridge National Lab. (ORNL), Oak Ridge, TN (United States). p. Medium: ED; Size: $56 \mathrm{p}$.

4. Pint, B.A., R. Pillai, M.J. Lance, and J.R. Keiser, Effect of Pressure and Thermal Cycling on Long-Term Oxidation in CO2 and Supercritical CO2, Oxid. of Met., 2020. 94(5-6), 505-526.

5. DeVan, J.H., Effect of alloying additions on corrosion behavior of nickel-molybdenum alloys in fused fluoride mixtures. 1969: United States.

6. Mccoy, H.E., R.L. Beatty, W.H. Cook, R.E. Gehlbach, C.R. Kennedy, J.W. Koger, A.P. Litman, C.E. Sessions, and J.R. Weir, New Developments in Materials for Molten-Salt Reactors, Nucl Appl Technol, 1970. 8(2), 156-\&.

7. Cho, H.S., J.W. Van Zee, S. Shimpalee, B.A. Tavakoli, J.W. Weidner, B.L. Garcia-Diaz, M.J. Martinez-Rodriguez, L. Olson, and J. Gray, Dimensionless Analysis for Predicting $\mathrm{Fe}-\mathrm{Ni}$ - $\mathrm{Cr}$ Alloy Corrosion in Molten Salt Systems for Concentrated Solar Power Systems, Corrosion, 2016. 72(6), 742-760.

8. Zheng, G., L. He, D. Carpenter, and K. Sridharan, Corrosion-induced microstructural developments in 316 stainless steel during exposure to molten Li2BeF4(FLiBe) salt, J. Nucl. Mater., 2016. 482, 147-155.

9. Mehrabadi, B.A.T., J.W. Weidner, B. Garcia-Diaz, M. Martinez-Rodriguez, L. Olson, and S. Shimpalee, Multidimensional Modeling of Nickel Alloy Corrosion inside High Temperature Molten Salt Systems, J. Electrochem. Soc., 2016. 163(14), C830-C838.

10. Pillai, R., S.S. Raiman, and B.A. Pint, First steps toward predicting corrosion behavior of structural materials in molten salts, J. Nucl. Mater., 2021. 546, 1.

11. Walker, M., D. Stapp, and J. Hinze, Collective Summary of sCO2 Materials Development Part II: High-Temperature Alloy Corrosion Behavior within Compact Heat Exchangers. 2019, ; Sandia National Lab. (SNL-CA), Livermore, CA (United States). p. Medium: ED; Size: 24 p.

12. Kung, S., J. Shingledecker, I.G. Wright, A.S. Sabau, B. Tossey, and T. Lolla, Corrosion of Heat Exchanger Alloys in Open-Fired $\mathrm{sCO}_{2}$ Power Cycles in The 6th International Supercritical CO2 Power Cycles Symposium. 2018: Pittsburgh, PA.

13. Pillai, R., H. Ackermann, and K. Lucka, Predicting the depletion of chromium in two high temperature Ni alloys, Corros. Sci., 2013. 69, 181-190.

14. Duan, R., A. Jalowicka, K. Unocic, B.A. Pint, P. Huczkowski, A. Chyrkin, D. Gruner, R. Pillai, and W.J. Quadakkers, Predicting Oxidation-Limited Lifetime of Thin-Walled Components of NiCrW Alloy 230, Oxidation of Metals, 2017. 87(1-2), 11-38.

15. Tucker, J., L. Árnadóttir, M. Anderson, B. Pint, Ö. Doğan, H. Saari, C. Jang, and S. Kung, Advancement of Supercritical Carbon Dioxide Technology through Round Robin Testing and Fundamental Modeling. 2019: United States. p. Medium: ED; Size: 85 p. 
16. Holcomb, G.R., C. Carney, and Ö.N. Doğan, Oxidation of alloys for energy applications in supercritical $C O<s u b>2</ s u b>$ and $H<s u b>2</ s u b>O$, Corros. Sci., 2016, Medium: ED; Size: p. 22-35.

17. Pint, B.A., Progression to Compatibility Evaluations in Flowing Molten Salts. 2020: United States. p. Medium: ED; Size: 73 p.

18. Pint, B.A. and J.R. Keiser, Supercritical CO2 Compatibility of Structural Alloys at 400-750 C. 2016, Oak Ridge National Lab.(ORNL), Oak Ridge, TN (United States).

19. Pint, B.A. and K.A. Unocic, The Effect of CO2 Pressure on Chromia Scale Microstructure at $750^{\circ} \mathrm{C}, \mathrm{JOM}, \mathbf{2 0 1 8}$. 70(8), 1511-1519.

20. Pint, B.A., S.S. Raiman, and J. Keiser, Lifetime modeling for a supercritical CO2-molten salt CSP power block. Journal Volume: 2126; Conference: SolarPACES 2018: International Conference on Concentrating Solar Power and Chemical Energy Systems - Casablanca, , Morocco - 10/2/2018 8:00:00 AM-10/5/2018 8:00:00 AM. 2019: ; Oak Ridge National Lab. (ORNL), Oak Ridge, TN (United States). Medium: ED; Size: 160005.

21. Deodeshmukh, V. and B.A. Pint, Long-Term Performance of High Temperature Alloys in Oxidizing Environments and Supercritical $C O<s u b>2</ s u b>$. Conference: Joint EPRI123HiMAT International Conference on Advances in High Temperature Materials 2019 , Nagasaki, Japan , 10/21/2019 8:00:00 AM-10/25/2019 8:00:00 AM. 2019: ; Oak Ridge National Lab. (ORNL), Oak Ridge, TN (United States). Medium: ED.

22. Lehmusto, J., J.M. Kurley, M.J. Lance, J.R. Keiser, and B.A. Pint, The Impact of Impurities on Alloy Behavior in Supercritical CO2 at 700 degrees C, Oxid. of Met., 2020. 94(1-2), 95-111.

23. Oleksak, R.P., G.R. Holcomb, C.S. Carney, L. Teeter, and Ö.N. Doğan, Effect of Surface Finish on High-Temperature Oxidation of Steels in $C O<s u b>2</ s u b>$, Supercritical $C O<s u b>2</ s u b>$, and Air, Oxid. of Met., 2019, Medium: ED; Size: p. 525-540.

24. Holcomb, G.R., C. Carney, and O.N. Dogan, Oxidation of alloys for energy applications in supercritical CO2 and H2O, Corros. Sci., 2016. 109, 22-35.

25. Oleksak, R.P., J.H. Tylczak, G.R. Holcomb, and Ö.N. Doğan, High temperature oxidation of Ni alloys in CO2 containing impurities, Corros. Sci., 2019. 157, 20-30.

26. Purgert, R., J. Shingledecker, J. Pschirer, R. Ganta, P. Weitzel, J. Sarver, B. Vitalis, M. Gagliano, G. Stanko, and P. Tortorelli, Boiler materials for ultra supercritical coal power plants. 2015, ; Energy Industries of Ohio, Independence, OH (United States). p. Medium: ED; Size: 1104 p.

27. Tortorelli, P.F., H. Wang, K.A. Unocic, M.L. Santella, and J.P. Shingledecker, Long-Term CreepRupture behavior of Inconel 740 and Haynes 282. Conference: Proceedings of the ASME Symposium on Elevated Temperature Application of Materials for Fossil, Nuclear, and Petrochemical Industries, Seattle, WA, USA, 20140325, 20140325. 2014: ; Oak Ridge National Lab. (ORNL), Oak Ridge, TN (United States). Medium: X.

28. Purohit, A. and W.F. Burke, Elevated temperature creep behavior of Inconel alloy 625. 1984: United States. p. 6.

29. Render, M., M.L. Santella, X. Chen, P.F. Tortorelli, and V. Cedro, Long-Term Creep-Rupture Behavior of Alloy Inconel 740/740H, Metallurgical and Materials Transactions A, 2021.

30. Pint, B.A., H. Wang, C.S. Hawkins, and K.A. Unocic, Technical Qualification of New Materials for High Efficiency Coal-Fired Boilers and Other Advanced FE Concepts: Haynes ${ }^{\circledR} 282 \AA A S M E$ Boiler and Pressure Vessel Code Case. 2020, ; Oak Ridge National Lab. (ORNL), Oak Ridge, TN (United States). p. Medium: ED; Size: 31 p.

31. Gomez-Vidal, J.C., Corrosion resistance of MCrAlX coatings in a molten chloride for thermal storage in concentrating solar power applications, npj Materials Degradation, 2017, Medium: ED; Size: Article No. 7.

32. Salinas-Solano, G., J. Porcayo-Calderon, J.G. Gonzalez-Rodriguez, V.M. Salinas-Bravo, J.A. Ascencio-Gutierrez, and L. Martinez-Gomez, High Temperature Corrosion of Inconel 600 in $\mathrm{NaCl-KCl} \mathrm{Molten} \mathrm{Salts,} \mathrm{Adv} \mathrm{Mater} \mathrm{Sci} \mathrm{Eng,} 2014$. 
33. Grégoire, B., C. Oskay, T. Meißner, and M. Galetz, Corrosion mechanisms of ferritic-martensitic P91 steel and Inconel 600 nickel-based alloy in molten chlorides. Part I: $\mathrm{NaCl-KCl} \mathrm{binary}$ system, Solar Energy Materials and Solar Cells, 2020. 215, 110659.

34. Wang, M.J., S. Zeng, H.H. Zhang, M. Zhu, C.X. Lei, and B.S. Li, Corrosion behaviors of 316 stainless steel and Inconel 625 alloy in chloride molten salts for solar energy storage, High Temperature Materials and Processes, 2020. 39, 340-350.

35. Sun, H., J. Wang, Z. Li, P. Zhang, and X. Su, Corrosion behavior of 316SS and Ni-based alloys in a ternary $\mathrm{NaCl}-\mathrm{KCl}-\mathrm{MgCl} 2$ molten salt, Solar Energy, 2018. 171, 320-329.

36. Pint, B.A., J.W. McMurray, A.W. Willoughby, J.M. Kurley, S.R. Pearson, M.J. Lance, D.N. Leonard, H.M. Meyer, J. Jun, S.S. Raiman, and R.T. Mayes, Re-establishing the paradigm for evaluating halide salt compatibility to study commercial chloride salts at $600^{\circ} \mathrm{C}-800^{\circ} \mathrm{C}$, Mater. Corr., 2019. 70(8), 1439-1449.

37. Render, M., M.L. Santella, X. Chen, P.F. Tortorelli, and V. III Cedro, Long-Term Creep-Rupture Behavior of Alloy Inconel 740 In preparation, 2021.

38. Maziasz, P.J., J.P. Shingledecker, N.D. Evans, Y. Yamamoto, K.L. More, R. Trejo, and E. LaraCurzio, Creep strength and microstructure of AL20-25+Nb alloy sheets and foils for advanced microturbine recuperators, J. Eng. Gas Turbines Power, 2007. 129(3), 798-805.

39. Purohit, A. and W.F. Burke, Elevated temperature creep behavior of Inconel alloy 625. Conference: Joint meeting of the American Nuclear Society and the Atomic Industrial Forum, Washington, DC, USA, 11 Nov 1984; Other Information: Portions are illegible in microfiche products. 1984: ; Argonne National Lab., IL (USA). Medium: ED; Size: Pages: 6.

40. Shingledecker, J.P., N.D. Evans, and G.M. Pharr, Influences of composition and grain size on creep-rupture behavior of Inconel (R) alloy 740, Mater. Sci. Eng., A, 2013. 578, 277-286.

41. Shingledecker, J.P. and G.M. Pharr, Testing and Analysis of Full-Scale Creep-Rupture Experiments on Inconel Alloy 740 Cold-Formed Tubing, J Mater Eng Perform, 2013. 22(2), 454462.

42. Goebel, H.H., G. Husmann, T. Voit, and M. Caliskan, Special Techniques in Diagnostic Myopathology, Turkish J Pediatr, 1994. 36(3), 223-237.

43. Tawancy, H.M., I.M. Allam, and N.M. Abbas, Effect of $\mathrm{Ni}_{3} \mathrm{Nb}$ Precipitation on the CorrosionResistance of Inconel Alloy 625, J. Mater. Sci. Lett., 1990. 9(3), 343-347.

44. Mathew, M.D., P. Parameswaran, and K. Bhanu Sankara Rao, Microstructural changes in alloy 625 during high temperature creep, Materials Characterization, 2008. 59(5), 508-513.

45. Duan, R., A. Jalowicka, K. Unocic, B.A. Pint, P. Huczkowski, A. Chyrkin, D. Grüner, R. Pillai, and W.J. Quadakkers, Predicting Oxidation-Limited Lifetime of Thin-Walled Components of $\mathrm{NiCrW}$ Alloy 230, Oxid. of Met., 2017. 87(1), 11-38.

46. Wagner, C., Theoretical Analysis of the Diffusion Processes Determining the Oxidation Rate of Alloys, J. Electrochem. Soc., 1952. 99(10), 369-380.

47. Pint, B.A., J.W. McMurray, A.W. Willoughby, J.M. Kurley, S.R. Pearson, M.J. Lance, D.N. Leonard, H.M. Meyer, J. Jun, S.S. Raiman, and R.T. Mayes, Re-establishing the paradigm for evaluating halide salt compatibility to study commercial chloride salts at $600^{\circ} \mathrm{C}-800^{\circ} \mathrm{C}$. https://doi.org/10.1002/maco.201810638(0).

48. Chyrkin, A., P. Huczkowski, V. Shemet, L. Singheiser, and W.J. Quadakkers, Sub-Scale Depletion and Enrichment Processes During High Temperature Oxidation of the Nickel Base Alloy 625 in the Temperature Range 900-1000 degrees C, Oxid. of Met., 2011. 75(3-4), 143-166.

49. Chyrkin, A., R. Pillai, H. Ackermann, H. Hattendorf, S. Richter, W. Nowak, D. Gruner, and W.J. Quadakkers, Modeling carbide dissolution in alloy 602 CA during high temperature oxidation, Corros. Sci., 2015. 96, 32-41.

50. Pillai, R., S.S. Raiman, and B.A. Pint, First steps toward predicting corrosion behavior of structural materials in molten salts, J. Nucl. Mater., 2021, Medium: ED; Size: Article No. 152755. 
51. Pillai, R., W.G. Sloof, A. Chyrkin, L. Singheiser, and W.J. Quadakkers, A new computational approach for modelling the microstructural evolution and residual lifetime assessment of MCrAlY coatings, Mat. at High Temp., 2015. 32(1-2), 57-67.

52. MobNi4, Thermo-Calc Software MobNi4/Ni-alloys mobility database version 4. 2018: Sweden.

53. Thermo-Calc, Thermo-Calc Software TCNi8/Ni-based superalloys database version 8. 2018 : Sweden.

54. Pillai, R., S.S. Raiman, D. Sulejmanovic, and B.A. Pint, Role of alloy chemistry in governing corrosion rates of candidate materials for molten salt reactors. 2022.

55. McLean, M. and B.F. Dyson, Modeling the effects of damage and microstructural evolution on the creep behavior of engineering alloys, J Eng Mater-T Asme, 2000. 122(3), 273-278.

56. Dyson, B.F. and S. Osgerby, Modeling Creep Corrosion Interactions in Nickel-Base Superalloys, Materials Science and Technology, 1987. 3(7), 545-553.

57. Dyson, B., Use of CDM in materials modeling and component creep life prediction, J Press VessT Asme, 2000. 122(3), 281-296.

58. Basoalto, H., S.K. Sondhi, B.F. Dyson, and M. McLean, A generic microstructure-explicit model of creep in nickel-base superalloys, Superalloys 2004, 2004, 897-906.

59. Pillai, R., Modelle zur Prognose der kriech- und oxidationsbedingten Lebensdauer thermisch belasteter Bauteile. 2015, RWTH Aachen University: Aachen. p. VII, 143 S. : Ill., graph. Darst.

60. Unocic, K.A., J.P. Shingledecker, and P.F. Tortorelli, Microstructural Changes in Inconel (R) 740 After Long-Term Aging in the Presence and Absence of Stress, Jom, 2014. 66(12), 25352542.

61. Hawk, J.A., T.L. Cheng, J.S. Sears, P.D. Jablonski, and Y.H. Wen, Gamma Prime Stability in Haynes 282: Theoretical and Experimental Considerations, J Mater Eng Perform, 2015. 24(11), 4171-4181.

62. Vattappara, K., Understanding the effect of temperature and time on Gamma prime coarsening for Nickel-base superalloy Haynes 282, in TRITA-ITM-EX. 2019. p. 71.

63. Tortorelli, P.F., H. Wang, K.A. Unocic, M.L. Santella, J.P. Shingledecker, and V. Cedro, III. in ASME 2014 Symposium on Elevated Temperature Application of Materials for Fossil, Nuclear, and Petrochemical Industries. 2014. 29-36.

64. de Oliveira, M.M., A.A. Couto, G.F.C. Almeida, D.A.P. Reis, N.B. de Lima, and R. Baldan, Mechanical Behavior of Inconel 625 at Elevated Temperatures, Metals-Basel, 2019. 9(3).

65. Ding, W., Y. Shi, F. Kessel, D. Koch, and T. Bauer, Characterization of corrosion resistance of $\mathrm{C} / \mathrm{C}-\mathrm{SiC}$ composite in molten chloride mixture $\mathrm{MgCl} 2 / \mathrm{NaCl} / \mathrm{KCl}$ at $700^{\circ} \mathrm{C}$, npj Materials Degradation, 2019. 3(1), 42.

66. Xi, J.Q., H. Jiang, C. Liu, D. Morgan, and I. Szlufarska, Corrosion of Si, C, and SiC in molten salt, Corros. Sci., 2019. 146, 1-9.

67. Walker, M., K.M. Armijo, J. Yellowhair, C.K. Ho, A. Bohinsky, J. Halfinger, and H. Feinroth, High Temperature Silicon Carbide Receiver Tubes for Concentrating Solar Power. 2019, ; Sandia National Lab. (SNL-CA), Livermore, CA (United States); Sandia National Lab. (SNLNM), Albuquerque, NM (United States). p. Medium: ED; Size: 82 p.

68. Sridharan, K., Corrosion of Structural Materials for Advanced Supercritical Carbon- Dioxide Brayton Cycle. 2017, ; Univ. of Wisconsin, Madison, WI (United States). p. Medium: ED; Size: $171 \mathrm{p}$.

69. Walker, M., W.L. York, J. Chames, J.D. Sugar, K. Frey, H. Feinroth, and E. Barringer, Silicon Carbide Multilayer Piping for 900oC Supercritical CO2 Brayton Cycle: Chemical Compatibility in CO2. 2019, ; Sandia National Lab. (SNL-CA), Livermore, CA (United States). p. Medium: ED; Size: 24 p.

70. Katoh, Y., L.L. Snead, I. Szlufarska, and W.J. Weber, Radiation effects in SiC for nuclear structural applications, Current Opinion in Solid State and Materials Science, 2012. 16(3), 143152.

71. Pint, B.A. and J.R. Keiser. 2014. 
72. Bremen, W., A. Naoumidis, and H. Nickel, Oxidation Behavior of Pyrolytically Deposited Beta Sic in Mixed Co-Co2 Atmosphere, J. Nucl. Mater., 1977. 71(1), 56-64.

73. Nagaoka, T., K. Kita, rsquo, ichiro, and N. Kondo, Hot corrosion of $A l<s u b>2</$ sub $>O<s u b>3</ s u b>$ and SiC ceramics by KCl\&ndash;NaCl molten salt, J Ceram Soc Jpn, 2015. 123(1440), 685-689.

74. Espinoza-Perez, L.J., S. Esquivel-Medina, and E. Lopez-Honorato, Influence of SiC microstructure on its corrosion behavior in molten FLiNaK salt, Ceram Int, 2021 . 47(11), 1552715532.

75. Gu, Y.F., J.X. Liu, Y. Wang, J.X. Xue, X.G. Wang, H.B. Zhang, F.F. Xu, and G.J. Zhang, Corrosion behavior of TiC-SiC composite ceramics in molten FLiNaK salt, Journal of the European Ceramic Society, 2017. 37(7), 2575-2582.

76. Opila, E.J. and Q.N. Nguyen, Oxidation of chemically-vapor-deposited silicon carbide in carbon dioxide, Journal of the American Ceramic Society, 1998. 81(7), 1949-1952.

77. Xue, W.D., X.M. Yang, J. Qiu, H.J. Liu, B. Zhao, H.H. Xia, X.T. Zhou, P. Huai, H.T. Liu, and J.Q. Wang, Effects of Cr3+ on the corrosion of SiC in LiF-NaF-KF molten salt, Corros. Sci., 2017. 114, 96-101.

78. Yang, X., D. Zhang, M. Liu, S. Feng, W. Xue, H. Liu, G. Yu, X. Zhou, H. Xia, P. Huai, Z. Li, Y. $\mathrm{Lu}, \mathrm{H}$. Zhou, and S. Dong, Corrosion of SiC induced by Hastelloy $N$ alloy and its corrosion products in LiF-NaF-KF molten salt, Corros. Sci., 2016, 62-67.

79. Lee, J.J., S.S. Raiman, Y. Katoh, T. Koyanagi, C.I. Contescu, X.X. Hu, and Y. Yang, Chemical compatibility of silicon carbide in molten fluoride salts for the fluoride salt-cooled high temperature reactor, J. Nucl. Mater., 2019. 524, 119-134.

80. Sorenson, B.A., D.L. Wyse, W.C. Koskinen, D.D. Buhler, W.E. Lueschen, and M.D. Jorgenson, Formation and Movement of C-14 Atrazine Degradation Products in a Sandy Loam Soil under Field Conditions, Weed Sci, 1993. 41(2), 239-245.

81. Walter, M.E. and G. Ravichandran, An Experimental Investigation of Damage Evolution in a Ceramic-Matrix Composite, J Eng Mater-T Asme, 1995. 117(1), 101-108.

82. Daniel, I.M. and G. Anastassopoulos, Failure Mechanisms and Damage Evolution in Crossply Ceramic-Matrix Composites, Int J Solids Struct, 1995. 32(3-4), 341-355.

83. Pickering, K.L., M.G.A. Efendy, and T.M. Le, A review of recent developments in natural fibre composites and their mechanical performance, Compos Part a-Appl S, 2016. 83, 98-112.

84. Tracy, J., Multi-scale Investigation of Damage Mechanisms in SiC/SiC Ceramic Matrix Composites, in Materials Science and Engineering. 2014, University of Michigan.

85. Pelleg, J., Creep in Silicon Carbide (SiC), in Creep in Ceramics. 2017, Springer International Publishing: Cham. p. 357-393.

86. Opila, E.J. and R.E. Hann, Paralinear oxidation of CVD SiC in water vapor, Journal of the American Ceramic Society, 1997. 80(1), 197-205.

87. Opila, E.J., Variation of the oxidation rate of silicon carbide with water-vapor pressure, Journal of the American Ceramic Society, 1999. 82(3), 625-636.

88. Parthasarathy, T.A., C.P. Przybyla, R.S. Hay, and M.K. Cinibulk, Modeling Environmental Degradation of SiC-Based Fibers, Journal of the American Ceramic Society, 2016. 99(5), 17251734.

89. Parthasarathy, T.A., R.A. Rapp, M. Opeka, and M.K. Cinibulk, Modeling Oxidation Kinetics of SiC-Containing Refractory Diborides, Journal of the American Ceramic Society, 2012. 95(1), 338-349.

90. Parthasarathy, T.A., R.A. Rapp, M. Opeka, and R.J. KeranS, A model for the oxidation of ZrB2, HfB2 and TiB2, Acta Mat., 2007. 55(17), 5999-6010.

91. Lamkin, M.A., F.L. Riley, and R.J. Fordham, Oxygen mobility in silicon dioxide and silicate glasses: a review, Journal of the European Ceramic Society, 1992. 10(5), 347-367.

92. Svehla, R.A., ESTIMATED VISCOSITIES AND THERMAL CONDUCTIVITIES OF GASES AT HIGH TEMPERATURES. 1962: United States. p. Medium: X; Size: Pages: 141. 
93. Pelleg, J., Diffusion in Silicon Carbide (Carborundum), in Diffusion in Ceramics. 2016, Springer International Publishing: Cham. p. 179-236.

94. Morscher, G.N. and J.D. Cawley, Intermediate temperature strength degradation in SiC/SiC composites, Journal of the European Ceramic Society, 2002. 22(14-15), 2777-2787.

95. Mital, S. and E.J. Pineda. 2018.

96. Lara-Curzio, E., Stress-rupture of Nicalon/SiC continuous fiber ceramic composites in air at 950 degrees C, Journal of the American Ceramic Society, 1997. 80(12), 3268-3272. 